\title{
The Rôle of the Surface Pretreatment in the Durability of Aluminium-Alloy Structural Adhesive Joints: Mechanisms of Failure
}

\author{
David J Bland ${ }^{1 *}$, Anthony J Kinloch ${ }^{1}$, John F Watts ${ }^{2}$ \\ ${ }^{1}$ Department of Mechanical Engineering \\ Imperial College London, Exhibition Road, London, SW72AZ, UK \\ ${ }^{2}$ Surrey Materials Institute, Faculty of Engineering and Physical Sciences \\ University of Surrey, Guildford, Surrey GU2 7XH, UK
}

\begin{abstract}
The durability of adhesively-bonded aluminium-alloy joints have been investigated by undertaking cyclic-fatigue tests in liquid water and in $55 \% \mathrm{RH}$, to establish the threshold adhesive fracture energy, $G_{\text {th }}$, below which crack growth will not occur. Three surface pretreatments were employed: grit-blast and degreasing (GBD), phosphoric-acid anodising (PAA) and PAA followed by the application of an anti-corrosion primer (PAAP). The ranking of the durability for the six systems, as assessed by the value of $\mathrm{G}_{\mathrm{th}}$, is as follows:

$$
\mathrm{PAAP}_{55 \% \mathrm{RH}} \cong \mathrm{PAA}_{55 \% \mathrm{RH}}>\mathrm{PAAP}_{\mathrm{H} 2 \mathrm{O}} \cong \mathrm{PAA}_{\mathrm{H} 2 \mathrm{O}}>\mathrm{GBD}_{55 \% \mathrm{RH}}>\mathrm{GBD}_{\mathrm{H} 2 \mathrm{O}}
$$

X-ray photoelectron spectroscopy, scanning electron microscopy and energy-filtered transmission-electron microscopy have been used to assess the nature of the locus of failure and the mechanisms of failure. For the GBD pretreated joints, tested in both the liquid water and in $55 \% \mathrm{RH}$ environments, failure occurs predominantly by the thermodynamic displacement of the adhesive from the metal substrate, according to the well-established principles of interfacial thermodynamics. In the case of the PAA and PAAP surface
\end{abstract}

\footnotetext{
* Present Address: VERTU, Fleet, Hants GU52 8DY, UK
} 
pretreatments, the anodising process provides additional adhesion forces and stability to the interface, which involves the formation of a microcomposite interphase region, and failure is shown to occur in a cohesive manner entirely within the adhesive for the tests undertaken in the $55 \% \mathrm{RH}$ environment. For the specimens tested in liquid water, again in the threshold region, the joints show loci of failure which are predominantly within the adhesive but with small areas of interfacial failure and oxide failure. The differences in durability for the PAAP and PAA when exposed to $55 \% \mathrm{RH}$ or liquid water is ascribed to the kinetics of aggregation of water in the environs of the metal/adhesive interface, this phenomenon occurring much more rapidly for exposure to liquid water during the cyclic-fatigue process than for the exposure to water vapour at $55 \% \mathrm{RH}$. A method based on the XPS analysis of failure surfaces has been used to assess the extent of interfacial failure of the joint prepared from anodised stock. This is shown to be up to $95 \%$ depending on treatment and exposure conditions.

Keywords: Adhesive bonding, aluminium, fracture mechanics, durability, XPS, locus of failure. 


\section{Introduction}

Adhesive bonding has many advantages over the more traditional approaches to the structural joining of materials, such as fusion-joining approaches via welding and brazing or mechanical-fastening methods via rivets. These include [1] the ability to join dissimilar materials with a uniform stress-distribution along the junction between the two substrates. In addition adhesive bonding lends itself readily to automated processing and can often provide the most appropriate, and cost effective, technology for the joining of a wide range of materials across a wide gamut of industries. In this respect, adhesive bonding is considered to be a mature joining process which, when considered at the design stage and used with the necessary design tools, provides a reliable, predictable and durable method for the joining process. It is for this reason that adhesive bonding is widely used in both the automotive and aerospace industries, where many aspects of the design- and buildprocess may be considered as safety critical. Notwithstanding, adhesive bonding is not without its problems and the foremost of these is the susceptibility of an adhesive joint, particularly involving metallic substrates, to undergo degradation on exposure to water when present either in the liquid or the vapour phase. In adverse situations this can seriously compromise the durability, and service-life, of adhesive joints in operation, since in many industrial applications adhesive joints are exposed to water and water vapour. This problem may be significantly accentuated if the adhesive joint is simultaneously subjected to cyclic-fatigue loading.

This phenomenon is well known by those responsible for the specification of adhesive-bonding procedures. Indeed, over the last four decades, or so, a range of approaches have been developed, often largely on an empirical basis, which ensure 
that adhesive joints are fit for purpose by possessing the required durability. Chief amongst these has been the development of a range of surface pretreatments that ensure the interface between the adhesive and the substrate remains relatively stable in the presence of aggressive environments, thus providing the necessary durability for the lifetime of the structure. In recent years there has been a steady contribution to the literature which relates performance, as exemplified by some form of accelerated test, to the mechanisms of failure, as deduced by a careful examination of the failure surfaces [2,3], or indeed intact interfaces examined as thin, electron transparent foils [3-5]. Surface analysis methods are employed to provide an accurate definition of the locus of joint failure and also to reveal the degradation of adhesive or substrate layer, and the aggregation of any species diagnostic of the exposure environment [6].

In the present paper we describe an investigation incorporating the dual approach described above. Using a fracture-mechanics methodology we have investigated the performance of adhesive joints manufactured using an aerospace aluminium-alloy (Grade: 2014A) and a hot-cured rubber-toughened epoxy adhesive. The aluminium-alloy substrates were pretreated in a variety of ways prior to being adhesively bonded and were tested under cyclic-fatigue loads in water: in either the vapour phase (i.e. $55 \% \mathrm{RH}$ ) or the liquid phase. The cyclic-fatigue tests were undertaken to obtain the relationship between the rate of crack growth rate per cycle, $\mathrm{da} / \mathrm{dN}$, and the maximum strain-energy release-rate, $\mathrm{G}_{\max }$, in the fatigue cycle. Double logarithmic plots of these data showed a threshold in the maximum value of $\mathrm{G}_{\max }$, below which crack growth did not occur. This threshold value, $\mathrm{G}_{\mathrm{th}}$, provides a figure of merit value for the performance of each set of pretreatment/environment 
variables that were studied. This is in accord with previous work [2,7], which revealed that such plots gave three clearly distinguishable regions:

(i) Region I which is a threshold region, and which is associated with low values of $d a / d N$ and $G_{\max }$, and where $G_{\max }=G_{t h}$.

(ii) Region II which is the linear portion.

(iii) Region III where the value of $G_{\max }$ starts to approach that of the adhesive fracture energy, $G_{c}$.

Following failure, the joints were examined using scanning electron microscopy and X-ray photoelectron spectroscopy, with the aim of elucidating the locus of failure and the degradation mechanisms responsible for joint failure. To assist in these studies, electron-transparent specimens were prepared from regions near the crack tip for examination using transmission electron microscopy (TEM), in conjunction with electron energy-loss spectroscopy, to provide energy-filtered TEM (EFTEM) images. 


\section{Experimental}

\subsection{Materials and Surface Pretreatment}

The aluminium alloy employed for this work was grade 2014A, a relatively high yieldstress and an unclad alloy. The composition of AA2014A is: $\mathrm{Cu} 4.5, \mathrm{Mn} 0.8, \mathrm{Mg} 0.5$, Fe 0.5 , Si 0.5 , Zn 0.25, Cr 0.1, balance Al. The material was supplied in plate form of thickness $9.75 \mathrm{~mm}$ and machined to give the tapered-double cantilever-beam (TDCB) geometry, as shown in Figure 1. The adhesive employed was a hot-cured rubber-toughened epoxy based upon a digycidyl ether of bisphenol A cured using dicyandiamide and supplied by Huntsman Advanced Materials, with a product designation of $X D^{\circledR} 4236-2$.

The aluminium-alloy substrates were treated by one of three pretreatment methods prior to bonding:

a) Degreased in acetone and boiling 1,1,1 trichloroethylene followed by gritblasting with $60 \mu \mathrm{m}$ alumina grit and then degreased again (referred to as GBD);

b) Following the pretreatment listed above in (a), phosphoric acid anodisation (PAA) was undertaken according to Boeing BAC 555 Specification [8];

c) The PAA pretreatment as described above in (b) was undertaken, plus the addition of a commercial corrosion-inhibiting primer, Cytec $\mathrm{BR}^{\circledR} 127$, to give a 
nominal thickness of the primer layer of nominally $2.5 \mu \mathrm{m}$ (with a maximum value of $7.5 \mu \mathrm{m}$ ) (referred to as PAAP).

\subsection{Manufacture of Adhesive Joints}

After manufacture and surface pretreatment of the aluminium-alloy beams (of length $310 \mathrm{~mm}$ ) two thin layers of poly(tetrafluoroethylene), typically extending around 60$80 \mathrm{~mm}$ from the loading end, were laid onto the substrate surface to create an initial crack during later testing. Meanwhile, the adhesive was degassed in a vacuum oven at $80^{\circ} \mathrm{C}$ to reduce its viscosity and enable it to wet the substrate surface more effectively. The thickness of the adhesive layer was controlled during manufacture using $0.5 \mathrm{~mm}$ wire inserts, one at either end of the TDCB joint. Finally, the joints were held in purpose-built jigs and were cured in an air-circulating oven at $130^{\circ} \mathrm{C}$ for three hours. They were then allowed to cool slowly to room temperature by switching off the oven overnight. Once ready, a thin coating of white spray-paint or liquid correction fluid was applied to the side of the specimen over the adhesive layer to facilitate taking visual crack-length readings. To further aid the measurement of the crack length, a scale of $1 \mathrm{~mm}$ spaced gridlines, printed onto paper, was attached to the side of the specimen, just below the lower bond-line, and the crack growth was monitored using a travelling microscope during the cyclic-fatigue tests. The geometry of the TDCB joint is shown schematically in Figure 1. 


\subsection{Fracture Mechanics Test Method}

The TDCB joint geometry was employed to determine the value of the adhesive fracture energy, $G_{c}$, following the standard test procedure $[9,10]$ at a test rate of 0.01 $\mathrm{mm} / \mathrm{min}$ and at $21 \pm 1^{\circ} \mathrm{C}$.

However, as well as being employed to determine the adhesive facture energy, $G_{c}$, the TDCB joint geometry was also used in the cyclic-fatigue studies to obtain the relationship between the rate of crack growth, da/dN, and the maximum strain-energy release-rate, $G_{\max }$, applied during a fatigue cycle $[11,12]$. These cyclicfatigue tests were conducted using a servo-hydraulic machine. The tests were conducted at $21^{\circ} \mathrm{C}$ in a tank of pure water or in an environmental cabinet containing a saturated solution of sodium nitrite which gave a measured $\mathrm{RH}$ of $55 \pm 5 \%$. In these tests, a sinusoidal loading waveform with a frequency of $5 \mathrm{~Hz}$ and a displacement ratio, $\mu_{\min } / \mu_{\max }$, of 0.5 was used. A range of maximum displacements, $\mu_{\max }$, was used to obtain the fatigue data over a wide range of values of the maximum strain-energy release-rate, $G_{\max }$, applied in a fatigue cycle. The value of $G_{\max }$ was calculated from the 'corrected beam theory' (CBT) method:

$$
G_{\max }=\frac{4 P_{\max }{ }^{2} m}{E_{s} B^{2}}\left[1+0.43\left(\frac{3}{m a}\right)^{\frac{1}{3}}\right]
$$

where $P_{\max }$ is the maximum load applied during the fatigue cycle, $\mathrm{m}$ is the geometry constant, see Figure $1, E_{s}$ is the modulus of the aluminium-alloy arms, $B$ is the arm 
thickness and $a$ is the crack length. It should be noted that the maximum strainenergy release-rate, $G_{\max }$, applied in the fatigue cycle has been employed in preference to the strain-energy release-rate amplitude $\left(\Delta G=G_{\max }-G_{\min }\right)$. This is because, during the unloading part of the fatigue cycle, the debonded surfaces typically came into contact, resulting in facial interference of the adhesive with itself (if cohesive failure in the adhesive occurs) or with the metal surface (if interfacial failure occurs). This has been observed to result in the generation of surface debris, which may prevent the crack from fully closing when it is unloaded, and hence may give an artificially high value of $G_{\text {min }}$. Thus, it has been suggested $[7,13,14]$ that it is better to use $G_{\max }$ instead of $\Delta G$, and this convention has been followed in the present studies.

The crack length, a, was measured either directly as function of the number of cycles, $\mathrm{N}$, with the aid of a travelling microscope, or theoretically ascertained by using the measured load-compliance data as a function of N. In the latter approach, then Equation 2 is the expression for the compliance, C, based on the CBT analysis [10]:

$$
C=\frac{8 m}{E_{s} B}\left[a+0.64\left(\frac{3}{m}\right)^{1 / 3} a^{2 / 3}-\frac{2}{3} x_{0}\right]
$$

where $x_{0}$ is the distance of the flat section of the beam profile from the loading end. From Equation 2 values of crack length may be readily determined from the measured compliance, and hence the crack length ascertained as a function of the number of fatigue cycles, $\mathrm{N}$. The method employed for obtaining values of the crack 
growth rate per cycle, da/dN, from the values of crack length, a, was the secant method as described in the relevant ASTM Standard [15] as:

$$
\frac{d a}{d \mathrm{~N}}=\frac{a_{i+1}-a_{i}}{\mathrm{~N}_{i+1}-\mathrm{N}}
$$

where $\mathrm{a}$ is the crack length and $\mathrm{N}$ is the number of fatigue cycles.

The fracture mechanics test data are the mean of several replicates, for the quasi-static tests six repeat specimens were used whilst for the fatigue tests duplicate specimens were tested, although one must recall that many individual data values are recorded during the crack growth studies. Thus the standard deviation values, obtained using standard statistical analysis, quoted for $G_{I C}$ and $G_{\text {th }}$ represent the scatter on the data. There is a potential for residual stresses to arise as a result of the adhesive cure schedule, but previous work [16] has shown that the effects of residual stresses on the value of $\mathrm{G}_{\mathrm{IC}}$ are not significant. For example, if such effects were considered for this type of adhesive then the value of $G_{I C}$ would be lower by no more than $1.5 \%$ of the calculated value. 


\subsection{Surface Analysis and Microscopy}

The failure surfaces of the joint following testing were examined using scanning electron microscopy (SEM) and X-ray photoelectron spectroscopy (XPS). SEM was carried out in the current work using either a Hitachi S-4000 microscope equipped with a field-emission source of a Hitachi S-3200N variable pressure microscope fitted with an energy-dispersive X-ray detector. Samples likely to exhibit electrostatic charging, such as the adhesive failure surfaces, were sputter-coated with a thin layer of gold prior to electron microscopy.

XPS analysis was undertaken using a Thermo Scientific Sigma Probe system. The spectrometer was operated in the constant analyser energy mode at a pass energy of $150 \mathrm{eV}$ for survey spectra) and $20 \mathrm{eV}$ or $50 \mathrm{eV}$ (for high resolution spectra depending on signal intensity). The Sigma Probe is equipped with a microfocus monochromatic AlKa X-ray source and was operated at a spot size of radius $50 \mu \mathrm{m}$. Quantitative surface analyses were calculated from peak areas of the high resolution spectra following the removal of a non-linear background and correction for analyser transmission.

In order to examine the adhesive/substrate interfacial region of a failed joint in more detail, electron transparent specimens were prepared from one side of a PAA treated aluminium-alloy TDCB adhesive joint. The failure surface was sputter-coated with gold and thin wedge-shaped strips were cut from this region of interest and mounted in a microtome capsule using an epoxy cold-setting resin. Samples were then cut by ultramicrotomy using the approach of Little et al. [3]. Transmission 
electron microscopy was undertaken using a Philips CM200 microscope equipped with parallel electron-energy loss (PEELS) spectroscopy for energy-filtered TEM (Gatan Imaging Filter [GIF]). The CM200 was operated using a $200 \mathrm{kV}$ beam in both dark-field and bright-field mode.

\section{Fracture Studies}

Adhesively-bonded TDCB joints were employed to obtain the values of fatigue crack growth rate, $\mathrm{da} / \mathrm{dN}$, and the maximum value of strain-energy release rate, $\mathrm{G}_{\max }$, applied in a fatigue cycle. The results are presented using double-logarithmic scales. The linear portion, Region II, was obtained using a least squares fit to the data.

As an example, Figure 2 shows the PAA pretreated aluminium-alloy TDCB joints tested at $55 \% \mathrm{RH}$ at $21 \pm 1^{\circ} \mathrm{C}$. Several noteworthy points emerge. Firstly, the values of the fatigue crack growth rate, da/dN, from both methods of assessment are in good agreement. Secondly, the fatigue curve shown in Figure 2 exhibits a similar characteristic sigmoidal shape obtained by many previous researchers, see for example $[3,7,13,14]$. Thirdly, there is a threshold in the maximum value for the applied strain-energy release-rate, $G_{t h}$, below which no significant fatigue crack growth occurred. Indeed, the data in this region showed that the values for da/dN were about $10^{-7} \mathrm{~mm} / \mathrm{cycle}$, and this meets the requirement for the value of da/dN to be considered negligible [15]. The value of $G_{t h}$ was found to be $225 \pm 9 \mathrm{Jm}^{-2}$. It is important to note that this is much lower than the value of adhesive fracture energy, $\mathrm{G}_{\mathrm{c}}$, ascertained under monotonically-loaded tests in the same test environment, which has a value of $850 \mathrm{Jm}^{-2}$ at $55 \% \mathrm{RH}$ at $21 \pm 1^{\circ} \mathrm{C}$. This confirms the damaging 
effects of the cyclic-fatigue tests compared with the monotonically-loaded tests. The importance of the threshold strain-energy release-rate, $\mathrm{G}_{\mathrm{th}}$, values is that they can be directly compared between joints, with differing surface pretreatments and environmental testing conditions, to rank the joints according to their relative longterm durability with respect to a given operating environment.

Another example is shown in Figure 3, which is a plot of the fatigue crack growth rate, da/dN, versus the maximum value of the strain-energy release-rate, $\mathrm{G}_{\max }$, applied in the fatigue cycle for the PAAP pretreated aluminium-alloy joints. These results were obtained at $21 \pm 1^{\circ} \mathrm{C}$ and at $55 \% \mathrm{RH}$. These data are directly compared with values for the PAA pretreated joints. As may be seen, there is no significant difference in the results from each of these different surface pretreatments in this environment.

The results from the cyclic-fatigue tests for the TDCB joints manufactured using the three different surface pretreatments tests and tested in the two different environments are all compared in Figure 4. As may be seen from these plots, values exist in all cases for a threshold in the maximum values of the strain-energy releaserate, $G_{t h}$, applied in the fatigue cycle, and these values are summarised in Table 1. The values of the resulting threshold values of the applied strain-energy release-rate, $\mathrm{G}_{\mathrm{th}}$, are given in Table 1. The ranking based upon the values of $\mathrm{G}_{\text {th }}$ of the various surface pretreatment/environment combination is also given in Table 1 and shows that:

$$
\mathrm{PAAP}_{55 \% \mathrm{RH}} \cong \mathrm{PAA}_{55 \% \mathrm{RH}}>\mathrm{PAAP}_{\mathrm{H} 2 \mathrm{O}} \cong \mathrm{PAA}_{\mathrm{H} 2 \mathrm{O}}>\mathrm{GBD}_{55 \% \mathrm{RH}}>\mathrm{GBD}_{\mathrm{H} 2 \mathrm{O}}
$$


The fundamental reasons why such a range in the values of $G_{\text {th }}$ are seen, and the underlying mechanisms which are responsible for these observations, are discussed below.

\section{Characteristics of the Failure Surfaces}

\subsection{Introduction}

In the present section the locus of failure of the various joints is identified using SEM, XPS and, in some cases, TEM/PEELS. The overall aim of this part of the investigation is the correlation of the durability of the adhesive joints with the characteristics of the failure surfaces. The analytical and microscopy data are presented below, and in the following section the durability and failure characteristics will both be considered to elucidate the mechanisms of failure.

\subsection{The GBD Surface Pretreatment}

The XPS data obtained from the GBD specimens when tested in $55 \% \mathrm{RH}$ and when immersed in water are presented in Table 2. Also included in this data set, for comparison, are the data from the control GBD surface (i.e. pretreated prior to bonding) and also the bulk (i.e. control) adhesive. SEM images of the unbonded

substrate surface and the substrate failure surfaces following environmental exposure are shown in Figure 5. The XPS survey spectra of the same surfaces, and that of the bulk adhesive, are shown in Figure 6. The similarity of the SEM images and the XPS survey spectra indicate that failure occurs predominantly at the 
adhesive/substrate interface with small amounts of failure in both the adhesive (as indicated by the presence of carbon and nitrogen on the metal failure surfaces although carbon is also observed on the surface of the unbonded surface as a result of the deposition of adventitious hydrocarbon contamination) and metal oxide regions (as confirmed by the presence of aluminium on the adhesive failure surface). By consideration of the concentration of carbon and nitrogen (on the metal failure surface relative to the control GBD substrate) and aluminium (on the adhesive) it is possible to estimate the proportions of interfacial failure and of cohesive failure within the adhesive and the oxide.

Thus, it can be estimated that for the $55 \% \mathrm{RH}$ exposure cyclic-fatigue tests some $75 \%$ of the failure is interfacial at the adhesive/metal oxide interface, whilst of the remaining cohesive failure about one-third occurs in the oxide and two-thirds in the adhesive. Hence, the locus of failure for the $55 \% \mathrm{RH}$ exposure can be defined as follows: interfacial 75\%; cohesive in the adhesive 17\%; cohesive in the metal oxide $8 \%$. In the case of the joints exposed to liquid water this becomes: interfacial $90 \%$; cohesive in the adhesive 5\%; cohesive in the metal oxide $5 \%$.

From Table 1, these results are in good accord with the $\mathrm{G}_{\mathrm{th}}$ data, which shows a significant reduction from $40 \mathrm{Jm}^{-2}$ for the $55 \% \mathrm{RH}$ tests to $24 \mathrm{Jm}^{-2}$ for the tests conducted in liquid water, with the concomitant increase in the amount of interfacial failure given above from $75 \%$ to $90 \%$. 


\subsection{The PAA Surface Pretreatment}

\subsection{1}

\section{Tests in Liquid Water}

SEM images of the unbonded PAA treated 'control' surface are presented in Figure 7, along with images of the metal and adhesive interfacial failure surfaces of joints taken from Regions I and II of the crack growth curve, see Section 1. The PAA pretreated surface of the aluminium-alloy shows the expected scalloping at low magnification (Figure 7a) and dimpling at higher magnification (Figure 7b).

The images taken from Region II of a joint test in water reproduce the scalloped morphology on the metal surface (Figure 7c) with the complementary morphology on the adhesive failure surface (Figure 7d). Thus, visually the locus of failure appears to be predominantly interfacial between the adhesive and the PAA generated oxide layer. The SEM images of the PAA pretreated surface of the aluminium alloy from Region I (Figures 7e \& f) shows a rather different morphology; and although the scalloped surface topography is evident in some regions there are clearly zones of retained adhesive residue on the metal substrate and evidence of yielding of this retained adhesive on the aluminium alloy.

Following the format of the GBD section above, the quantitative XPS data for the PAA substrates are presented in Table 3 for the joints exposed to liquid water in the fast crack growth regimes (Region II), and Table 4 for the slow crack growth regimes, near to the threshold value, $\mathrm{G}_{\mathrm{th}}$, (Region I) in water. XPS survey spectra 
are presented in Figure 8 which compare the characteristics of the unbonded PAA substrate with that of the substrate failure surface from Region I of the crack growth curve when tested in water. As before, it is possible to estimate the proportion of failure types from the XPS data and for the tests carried out in water, the Region II failure is predominantly interfacial with only a very small concentration of aluminium retained on the adhesive side of the joint. Bearing in mind the well known morphology of the PAA pretreatment with fibrils of oxide observed at the surface of the porous columnar oxide structure, which has been confirmed by the TEM data presented below, it is quite likely that that this relatively weak aluminium signal is a result of the fracture of such features which now protrude from, or lie flat with, the adhesive failure surface. From the nature of the cyclic-fatigue test, where the failure surfaces are repetitively open and closed on each other, then this seems to be a likely explanation. However, for the very slow fatigue crack-growth regime (Region I), it is clear that the locus of failure has moved away from the interface and into the adhesive itself. Some $90 \%$ of the failure occurred cohesively within the adhesive layer, with approximately only $10 \%$ occurring along the interface or within the oxide layer itself. (Further support for the small amount of oxide in the adhesive failure XPS analysis resulting from a combination of oxide fracture and interfacial failure will be given in $\$ 5.4$.

TEM of the intact interface is presented in Figure 9, which shows the morphology and the formation of an inter-diffusion zone between the oxide and the adhesive, sometimes referred to as a 'micro-composite interphase'. The region ahead of the crack tip (i.e. the unfailed region of the joint) from a sample prepared from a cyclic-fatigue test undertaken in water is shown in Figure 10. This sample is 
taken from a TDCB test piece which had been tested for a number of weeks and reached its threshold crack-growth zone.

\subsubsection{Tests in $\mathbf{5 5 \%} \mathrm{RH}$}

Turning now to the cyclic-fatigue tests on the bonded TDCB joints prepared using the PAA pretreatment and undertaken in the $55 \% \mathrm{RH}$ environment, the SEM images indicate that cohesive failure within the adhesive occurs with the failure surfaces showing striation lines which are indicative of the direction of crack growth. This is reflected in the XPS analyses reported for the failure surfaces of Region II of the crack growth curve in $55 \% \mathrm{RH}$, the two analyses are remarkably similar in terms of both the major (carbon and oxygen) and minor (nitrogen, silicon and chlorine) elements detected. Thus, both the SEM and XPS results indicated cohesive failure in the adhesive layer for the $55 \% \mathrm{RH}$ tests.

\subsection{The PAAP Surface Pretreatment}

\subsection{1 \\ Tests in Liquid Water}

The PAAP surface pretreatment is based on the PAA process considered above but with the additional application of a commercial corrosion-inhibiting primer. The primer is applied to a nominal thickness of $2.5 \mu \mathrm{m}$ which is sufficient to obscure the characteristic PAA surface topography, as shown in Figure 11. The SEM images from the apparently interfacial failure surfaces of a specimen tested in water and taken from the cyclic-fatigue threshold growth zone are shown in Figure 12. The 
metal side of the joint, see Figure $12 \mathrm{a}$, shows a significant amount of retained adhesive and material which seems to have exhibit a "mud-cracking" pattern, which may provide a clue to the degradation mechanism. The adhesive side of the failure surface shows very few distinctive features, apart from well-defined rectangular craters which are thought to result from oxide regions that have undergone pull-out on failure.

The quantitative surface chemical analyses obtained from the XPS data for the PAAP control and joints tested in water are presented in Table 4. XPS survey spectra for the control PAAP surface and the failure surfaces from a joint tested in water are presented in Figure 13. These data indicate that the failures are predominantly cohesive within the organic phase, with less that $5 \%$ of the locus of failure occurring in the oxide or at the oxide/polymer interface.

The metal-substrate side of the failed joint was gold-coated and carefully sectioned to provide a sample for TEM studies. The bright field image of Figure 14a shows (from right to left) metal, oxide and what appear to be adhesive regions. This interpretation is confirmed by the EFTEM elemental images of Figure $14 \mathrm{~b}$ which shows the usual intermixing of organic and inorganic phase to give a 'microcomposite' interphase region, but with a residual layer of some $50-100 \mathrm{~nm}$ of primer or adhesive remaining on the anodised-oxide layer. 


\subsubsection{Tests in $\mathbf{5 5 \%} \mathbf{R H}$}

The XPS and SEM results for PAAP joints exposed to $55 \% \mathrm{RH}$ are not included as the results are very similar to those for the PAA surface pretreatment with the locus of failure occurring cohesively within the adhesive layer, well away from the substrate/adhesive interface. In the case of the PAAP pretreatment the difference between the locus of failure for tests carried out in $55 \% \mathrm{RH}$ and water is not so stark as for the PAA pretreatment.

\subsection{Summary}

This section has described the interfacial characteristics of the adhesive joints and presented SEM images and surface analysis data obtained from the interfacial failure surfaces of joints exposed to either $55 \% \mathrm{RH}$ of water. In the case of the cyclicfatigue tests conducted in the liquid water environment, it is shown that variations in the locus of failure can occur depending on whether specimens were taken from Region I or Region II of the crack growth curve. It is helpful to bring the XPS based assessments of the locus of failure together at this point and this is done in Table 6 . This clearly identifies the pretreatments that provide good durability as having a locus of failure of greater than $90 \%$ cohesive failure in the adhesive.

TEM and EFTEM have been used to assess the condition of the dry PAA substrate/adhesive interface and the region just ahead of the crack tip following water exposure. In addition it has proved possible to prepare a TEM specimen from 
the metal fracture surface of a PAAP pretreated aluminium-alloy joint and visualise a very thin layer of the primer/adhesive phase.

\section{Loci of Failure and Mechanisms of Environmental Attack}

\section{$5.1 \quad$ Introduction}

The aim of the present section is to consider the analytical data of $\S 4$ and establish if there are clear correlations with the fracture mechanics durability data of $\S 3$, both in terms of the locus of failure that is observed and the mechanism of environmental attack that is considered to be responsible for failure. The summary of Table 1 indicates the ranking of the various pretreament/environment combinations, and provides useful data for this discussion, clearly showing that the six combinations fall into three groups of two. For this reason failure mechanisms with be considered for (a) the two GBD environments together, (b) then the PAA and PAAP pretreatments will be considered for the tests conducted in $55 \% \mathrm{RH}$ and (c) then finally the PAA and PAAP pretreatments for the tests conducted in liquid water.

\subsection{The GBD Surface Pretreated Joints}

In the ranking order, see Table 1 , of the six combinations of pretreatment and environments the GBD pretreatment performed, as expected, very poorly with the lowest rankings for the performance of such joints when exposed to $55 \% \mathrm{RH}\left(\mathrm{G}_{\mathrm{th}}=\right.$ $\left.40 \mathrm{Jm}^{-2}\right)$ and upon exposure to liquid water $\left(G_{\mathrm{th}}=24 \mathrm{Jm}^{-2}\right)$. Turning to the locus of 
failure data of Table 6 , it can be seen that both loci of failures are predominantly interfacial with humid and wet environments having $76 \%$ and $90 \%$ interfacial failure, respectively. Interfacial failure following environmental exposure is often associated with the thermodynamic displacement of the adhesive from the metal oxide of the substrate $[1-3,17]$. The water, present at the interface, has a higher affinity for the high surface-energy oxide than the adhesive does and the latter is displaced from the oxide surface by the water. This phenomenon is well known $[3,17]$ and is often cited as the cause of joint failure on exposure to water, particularly if no load, or a stress, well below the yield stress, is applied. The surface thermodynamics, although indicating that failure is energetically favourable, give no clue as to the kinetics of the situation and it is not unusual for such a thermodynamic displacement mechanism to be in competition with other mechanisms of environmental attack, such as degradation of the adhesive (sometimes referred to as plasticisation) close to the interface. In such situations where the interface is very stable, interfacial failure may not even be observed as the kinetics of the process are much slower than competing processes. In the current work the situation has been exacerbated by the cyclicfatigue loading, which allows ready access of both vapour and condensed water to the crack tip. This has led to failures at very low adhesive fracture energies and the associated prevalence of interfacial failure, the liquid water exposure being somewhat more aggressive than the humid environment. This is presumably a result of the greater flux of water molecules seen by the interfacial region of the adhesive joint. 


\subsection{The PAA and PAAP Surface Pretreated Joints in $55 \% \mathrm{RH}$}

In consideration of both the phosphoric-acid anodising pretreatments (i.e. PAA and PAAP), the dominant factor is the test environment with those tested in water giving significantly poorer performance that those tested in a humid atmosphere, as shown in Table 1 in §3. Of particular significance is the lack of influence of the primer on the threshold fracture energy, $G_{\text {th }}$, for a given environment. When the loci of failure of the PAA and PAAP pretreatments are considered, both pretreatments reveal the a very similar fracture path in the bulk of the adhesive, well removed from the substrate/adhesive interface. The conclusion from this is clear: the micro-porous nature of the anodised substrate gives rise to the expected micro-composite interphase region [3] between the anodic-oxide layer and the adhesive, or primer, and this is interphase is sufficiently durable not to be adversely affected by the cyclic-fatigue loading in $55 \% \mathrm{RH}$. Thus, the damage accumulates in the adhesive, and cohesive failure of the adhesive layer is eventually observed in the cyclic-fatigue tests undertaken in this environment.

\subsection{The PAA and PAAP Surface Pretreated Joints in Liquid Water}

For the PAA and PAAP pretreated joints tested in liquid water it is clear that the results fall midway between the GBD pretreated joints in either environment and the PAA, and PAAP, pretreated joints tested in the $55 \% \mathrm{RH}$ environment. In the case of the PAA pretreatment the failure at a rapid crack growth rate (i.e. Region II) is associated with interfacial failure indicating that the water has ample access to the 
crack tip and the conjoint action of stress and liquid water is able to enhance the displacement of the adhesive from the oxide surface, although a small amount of adhesive remains on the metal substrate. This fact is reflected in the surface concentration of carbon which is $38 \%$, compared with $16 \%$ for the freshly anodised substrate. At a slower crack growth rate (i.e. Region I, which defines $G_{t h}$ ) a significant amount of cohesive failure in the adhesive layer is seen and this represents the predominant locus of failure (>90\%). It would seem that, at the slower crack growth rates, water is able to do more damage to the adhesive itself and failure occurs in the adhesive, but very close to the interface. The locus of failure for the PAA pretreatment appears to move from the bulk adhesive to the adhesive interface as the crack-growth rate increases (i.e. from Region I to II), which appears counter-intuitive. However, it is thought to relate to the manner in which the interface, the well-defined plane between adhesive and substrate, responds to stress and water; which is in a rapid and catastrophic manner whilst the damaging effect of water alone is a much slower process as observed in the Region I failures.

At this point it is helpful to turn to the schematic representation of the morphology of the PAA pretreatment provided by Venables [18], see Figure 15, and explore the area occupied by each phase (i.e. the adhesive, oxide "hexagonal structure" and oxide "fingers"). Thus, the description of any interfacial failure is clearly somewhat simplistic since the microstructure of the interfacial region is composed of (a) a micro-composite array of adhesive penetrated into the oxide matrix and (b) of the interface between the adhesive and the oxide. Such a microstructure of the interfacial region is clearly seen in the cross-sectional TEM 
images of Figures 9 and 10; and the dimensions are consistent with those observed by Venables (18), see Figure 15.

Assuming the hexagonal structure has a length of side of $40 \mathrm{~nm}$ and a thickness of $10 \mathrm{~nm}$ (as do the oxide fingers) and that each wall is shared between two cells and each finger between three cells if can be shown that, at a plane at the top of the porous oxide structure in an adhesive joint, $70 \%$ of the area is adhesive, $25 \%$ oxide of the porous hexagonal structure and $5 \%$ the area of the oxide fingers. Returning now to the data for Region II of the PAA joints tested in water (Table 3), it is possible to confirm the locus of failure given in Table 6. The XPS analysis of the control surface gives $19 \%$ aluminium but the metal failure surface in water is ca. $12 \%$. This is consistent with an interfacial failure along the tops of the oxide wall, with some residual adhesive left in the pores (but with the adhesive not failing at the top of all of the pores, as the concentration of aluminium would then be significantly lower: i.e. $0.25 \times 18=4.5 \%$ ). Hence, a great deal of the inner surface of the pores is exposed to increase the concentration of aluminium to $12 \%$. Thus, the $95 \%$ interfacial failure stated in Table 6 reflects oxide/adhesive failure at the plane of the cell tops with a significant amount of adhesive failure within the cellular structure. The extent to which failure occurs near the top or the bottom of the cells is open to speculation, although for more than half of the cells no adhesive is discernible by XPS. For the adhesive side the situation is much more straightforward. The $0.9 \%$ aluminium is the amount predicted by the area occupied by the oxide "fingers" $(0.05$ $x 18 \%)$ and this assay of aluminium at the adhesive failure surface is consistent with the area in the adhesive occupied by fractured oxide fingers indicating failure of these oxide protrusions at a plane at the top of the porous oxide structure. 
Using the same approach for the threshold, Region I, failure data of the PAA pretreated joints of Table 4, then the adhesive failure surface yields a very similar XPS analysis of Region II indicating failure of the oxide protrusions. The metal surface, however, has a significantly lower surface concentration of aluminium $(5.7 \%)$ than the Region II failure, indicating the failure is now predominantly cohesive within the adhesive with a small (some 25 area \%) being interfacial or cohesive in the oxide, plus the area of $5 \%$ that accounts for the fracture of the oxide protrusions.

Turning now to the PAAP XPS results, these indicate that the failure is once again concentrated in the organic phase, with very small amounts of aluminium oxide being observed on both failure surfaces. In principle this could be a result of small amounts of interfacial failure or cohesive failure of the oxide protrusions in the adhesive layer. Bearing in mind the TEM data of Figure 14, the latter explanation is considered more likely. Further, consideration of the EFTEM of Figure 14 establishes that there is an organic layer of some $50-100 \mathrm{~nm}$ on the anodic oxide itself. Bearing in mind that the primer was applied to a nominal thickness of $2.5 \mu \mathrm{m}$, this would tend to indicate that failure was associated with the primer layer. The concentration of nitrogen in the PAAP surface analysis of both sides of the PAAP failures that occur in water are significantly higher that both the unbonded PAAP substrate and also the adhesive control results (Table 2). This indicates the interdiffusion of the primer and the adhesive in general but more specifically the dicyandiamide curing agent. It is perhaps surprising that the application of a primer to the PAA substrate leads to a rather modest improvement in threshold strain-energy release rate (a $G_{t h}$ value of $112 \mathrm{Jm}^{-2}$ c.f. $100 \mathrm{Jm}^{-2}$ for the unprimed PAA substrate). Previous work with both 
epoxy and organosilane primers has shown their efficacy in improving bond durability. In the case of a water-borne epoxy primer used with a dry film adhesive it has been shown that the primer is required to ensure the successful penetration of the organic phase into the porous PAA structure [3]. The efficient use of an organosilane primer requires the formation of discrete chemical (covalent) bonds at the interface which are very resistant to hydrodynamic attack $[19,20]$. In the current work the primer employed is specified as a corrosion-resistant primer and as such will be loaded with sparingly soluble pigmentation (in this case strontium chromate) designed to slowly release species $\mathrm{Cr}(\mathrm{VI})$ that will migrate to the interface and inhibit the corrosion process. These processes are very slow and are unlikely to have been activated during the cyclic-fatigue tests, as evidenced by the absence of the active ions in the surface analysis of either the PAAP unbonded surface or the failure surfaces.

\subsection{Summary}

The commentary above reinforces the well-established notion that it is the stability of the adhesive/substrate interface that is the all-important feature in the durability of adhesive joints. This work shows that "stability" is not only a reflection on the thermodynamic nature of the interface itself but also on the nature of the exposure medium (liquid or vapour), which in turn influences the kinetics of arrival of the water molecules at the region of the interphase.

Reviewing the ranking order of Table 1, for the threshold Region I, shows that $55 \% \mathrm{RH}$ exposure is relatively benign compared with liquid water and that the 
pretreatments rank in the order of PAAP $\geq$ PAA $>$ GBD. The respective loci of failure range from predominantly cohesive in the adhesive for both the PAA and PAAP pretreatments but to predominantly interfacial for the GBD pretreatment. Several interesting points arise.

Firstly, the failure of the joints subjected to the GBD treatment is of the hydrodynamic displacement type discussed by many workers in the past and can thus be understood by considering the thermodynamics of the substrate/adhesive/water system [3, 17].

Secondly, the anodised pretreatments show relatively little difference between the primed and unprimed substrates but a significant change in terms of the nature of the environment to which they have been exposed. The similarity of response from the two substrate types is perhaps unsurprising and has been dealt with in the previous section. It is thus appropriate to consider the kinetics of water diffusion that bring about degradation in the environs of the interface, but predominantly in the adhesive. In the case of $55 \% \mathrm{RH}$ the partial pressure of water is low with both failures occurring within the adhesive. In the case of liquid water there is a ready supply of water molecules which, exacerbated by the cyclic nature of the test means the crack tip sees a small amount of interfacial failure, with, of course, the fracture of the oxide fingers extending into the adhesive $(<5 \%$ in the case of the PAAP treatment and a maximum of $10 \%$ for the PAA). It is interesting to note that, although there is a small but significant difference in the loci of failure, this has a disproportionate effect on the durability, for example compare the results shown in Tables 1 and 6 . This might be due solely to the effect of the small extent of interface 
failure that occurs when a liquid water, as opposed to a $55 \% \mathrm{RH}$, environment is used; but it might be due to exposure to liquid water also leading to degradation of the adhesive.

Finally, therefore, in assessing the behaviour of an adhesive bonding system in the presence of water one must consider the response of the adhesive to water in both physical states.

\section{Conclusions}

The durability of adhesively-bonded aluminium-alloy joints have been investigated by undertaking cyclic-fatigue tests in liquid water and in $55 \% \mathrm{RH}$, to establish the threshold adhesive fracture energy, $G_{\text {th }}$, below which crack growth will not occur. Three surface pretreatments were employed: grit-blast and degreasing (GBD), phosphoric-acid anodising (PAA) and PAA followed by the application of an anticorrosion primer (PAAP). The ranking of durability for the six systems, as assessed by the value of $G_{t h}$ for the threshold Region I, is as follows:

$$
\mathrm{PAAP}_{55 \% \mathrm{RH}} \cong \mathrm{PAA}_{55 \% \mathrm{RH}}>\mathrm{PAAP}_{\mathrm{H} 2 \mathrm{O}} \cong \mathrm{PAA}_{\mathrm{H} 2 \mathrm{O}}>\mathrm{GBD}_{55 \% \mathrm{RH}}>\mathrm{GBD}_{\mathrm{H} 2 \mathrm{O}}
$$

The loci of joint failure for the GBD joints are predominately interfacial with failure being ascribed to the thermodynamic displacement of the adhesive by water molecules present at the interface.

In the case of the phosphoric-acid anodised (PAA) substrates the porous nature of the pretreatment gives rise to a microcomposite interphase region which leads to joints prepared using this pretreatment exhibiting relatively good durability 
on exposure to both water and water vapour. By consideration of the morphology of such an interphase it has proved possible to develop a model in which failure of the oxide protrusions and a true interfacial failure between the porous oxide morphology and the adhesive can be identified. The addition of a primer layer appears to have no significant beneficial effect on the durability of the joints. A combination of XPS and EFTEM indicates that the failure of such joints are purely cohesive in the adhesive for exposure of the joints to water vapour. However, when the tests are conducted in liquid water then a small extent of interfacial failure is observed for both pretreatments when tested at the threshold region of the fatigue curve, where the value $G_{t h}$ of is ascertained. The small changes in the loci of failure for the PAA and PAAP joints which results from water immersion indicates that degradation of the interfacial adhesion is responsible for the inferior durability of the joints in the liquid water environment.

\section{Acknowledgments}

DJB thanks the EPSRC for provision of a studentship.

\section{References}

1. Kinloch A J, Proc Inst Mech Eng, 221 (Part G), 307, (1997).

2. Kinloch A J, Korenberg C F, Tan K T, Watts J F, J Mater Sci, 42, 6353, (2007).

3. Kinloch A J, Little M S G, Watts J F, Acta Materialia, 48, 4543, (2000). 
4. Bland D J, Kinloch A J, Stolojan V, Watts J F, Surf Interf Anal, 40, 128, (2008).

5. Bertho J, Stolojan V, Abel M-L, Watts J F, Micron, 41, 130, (2010).

6. Watts J F in "Shreir's Corrosion" Vol 3, Ed Richardson J A, Elsevier BV, 2010, pp $2463-2481$.

7. Jethwa J K, Kinloch A J, J Adhes, 61, 71, (1997).

8. Boeing BAC 5555 Specification

9. ISO Standard, 25217, (2009).

10. Blackman B K R, Hadavania H, Kinloch A J, Paraschi M, Williams J G, Eng Fract Mech, 70, 233, (2002).

11. Fernando M, Harjoprayitno W W, Kinloch A J, Int J Adhes Adhes, 16, 113, (1996).

12. Comyn J, in Durability of Structural Adhesives, Ed Kinloch A J, Applied Science Publishers, London, 1983, p 85.

13. Little M S G, PhD Thesis University of London, 1999

14. Tan K T, PhD Thesis University of London, 2005.

15. ASTM Standard E647:2005.

16. Blackman B K R, Kinloch A J, Rodriguez Samchez S, Teo W S, Williams J G, Eng Fract Mech, 76, 2868, (2009).

17. Gledhill R A, Kinloch A J, J Adhes, 6, 315, (1974).

18. Venables J A, J Mater Sci, 19, 2431, (1984).

19. Abel M-L in Handbook of Adhesion Technology, Eds da Silva L F M, Öchsner A, Adams R D, Springer-Verlag Berlin Heidelberg, (2011), p $237-258$

20. Sautrot M, Abel M-L, Watts J F, Powell J, J Adhes, 81, 163, (2005). 
Table 1. Threshold strain-energy release-rate, $G_{\mathrm{th}}$, for GBD, PAA and PAAP surface pretreatments acting as a method of ranking.

\begin{tabular}{|c|c|c|c|}
\hline Surface Pretreatment & Testing Conditions & $\mathrm{G}_{\mathrm{th}}\left[\mathrm{Jm}^{-2}\right]$ & Ranking \\
\hline PAAP & $55 \% \mathrm{RH}$ & $225 \pm 9$ & $1=$ \\
\hline PAA & $55 \% \mathrm{RH}$ & $225 \pm 9$ & $1=$ \\
\hline PAAP & Water & $112 \pm 6$ & $2=$ \\
\hline PAA & Water & $100 \pm 7$ & $2=$ \\
\hline GBD & $55 \% \mathrm{RH}$ & $40 \pm 4$ & 3 \\
\hline GBD & Water & $24 \pm 4$ & 4 \\
\hline
\end{tabular}

Table 2. Quantitative surface analysis for failure surfaces of joints prepared from degreased and grit blasted (GBD) aluminium-alloy substrates. Analyses are for unbonded surfaces and joints exposed to $55 \% \mathrm{RH}$ and liquid water.

\begin{tabular}{|l|c|c|c|c|c|c|c|c|c|}
\hline \multirow{2}{*}{ Specimen } & \multicolumn{7}{|c|}{ Surface composition/atomic \% } \\
\cline { 2 - 11 } & $\mathrm{C}$ & $\mathrm{O}$ & $\mathrm{Al}$ & $\mathrm{Mg}$ & $\mathrm{N}$ & $\mathrm{Ca}$ & $\mathrm{Na}$ & $\mathrm{Si}$ & $\mathrm{Cl}$ \\
\hline Control Al GBD & 33.8 & 46.3 & 15.7 & trace & trace & 0 & 3.1 & 1.1 & 0 \\
\hline Control Adhesive & 81.5 & 12.4 & 0 & 0 & 1.4 & 0 & 0 & 4.7 & trace \\
\hline $55 \% \mathrm{RH}$; Metal Side & 43.6 & 38.6 & 12.5 & 1.7 & 1.0 & 0.7 & 0.7 & 1.2 & 0 \\
\hline 55\% RH; Adhesive & 75.3 & 20.6 & 1.2 & 0 & 2.1 & 0 & 0 & 0.5 & 0.3 \\
\hline Water; Metal Side & 38.5 & 44.8 & 12.5 & 0.8 & 1.4 & 0.7 & 0 & 1.3 & 0 \\
\hline Water; Adhesive & 74.3 & 20.3 & 0.6 & 0 & 3.2 & 0.8 & 0.6 & 0 & 0.2 \\
\hline
\end{tabular}


Table 3. Quantitative surface analysis for failure surfaces of joints prepared from phosphoric-acid anodised (PAA) aluminium-alloy substrates. Analyses are for the unbonded surface and joints exposed to $55 \% \mathrm{RH}$ and to liquid water, in Region II of the crack growth curve.

\begin{tabular}{|l|c|c|c|c|c|c|c|c|c|c|}
\hline \multirow{2}{*}{ Specimen } & \multicolumn{9}{|c|}{ Surface composition/atomic \% } \\
\cline { 2 - 12 } & $\mathrm{C}$ & $\mathrm{O}$ & $\mathrm{Al}$ & $\mathrm{Mg}$ & $\mathrm{N}$ & $\mathrm{Ca}$ & $\mathrm{Na}$ & $\mathrm{Si}$ & $\mathrm{Cl}$ & $\mathrm{P}$ \\
\hline Control Al PAA & 16.0 & 61.8 & 18.7 & 0 & 1.2 & trace & 0 & 0.8 & 0 & 1.5 \\
\hline $55 \% \mathrm{RH}$; Metal Side & 79.2 & 15.9 & 0 & 0 & 1.3 & 0 & 0 & 3.6 & trace & 0 \\
\hline $55 \% \mathrm{RH}$; Adhesive & 81.3 & 14.1 & 0 & 0 & 1.2 & 0 & 0 & 3.4 & trace & 0 \\
\hline Water; Metal Side & 43.7 & 39.4 & 12.3 & 0.9 & 1.6 & trace & trace & 1.6 & 0 & 0.5 \\
\hline Water; Adhesive & 78.0 & 16.8 & 0.9 & trace & 1.1 & 0.5 & 0.5 & 2.2 & 0 & tr \\
\hline
\end{tabular}

Table 4. Quantitative surface analysis for failure surfaces of joints prepared from phosphoric-acid anodised (PAA) aluminium-alloy substrates. Analyses are for joints exposed to liquid water, in Region I of the crack growth curve.

\begin{tabular}{|l|c|c|c|c|c|c|c|c|c|c|}
\hline \multirow{2}{*}{ Specimen } & \multicolumn{9}{|c|}{ Surface composition/atomic \% } \\
\cline { 2 - 12 } & $\mathrm{C}$ & $\mathrm{O}$ & $\mathrm{Al}$ & $\mathrm{Mg}$ & $\mathrm{N}$ & $\mathrm{Ca}$ & $\mathrm{Na}$ & $\mathrm{Si}$ & $\mathrm{Cl}$ & $\mathrm{P}$ \\
\hline Water; Metal Side & 53.8 & 29.8 & 5.7 & trace & 3.6 & 1.2 & trace & 2.8 & 3.1 & trace \\
\hline Water; Adhesive & 79.6 & 15.2 & 0.8 & 0.4 & 1.1 & 0.6 & 0.6 & 1.7 & 0 & trace \\
\hline
\end{tabular}


Table 5. Quantitative surface analysis for failure surfaces of joints prepared from phosphoric-acid anodised aluminium substrates treated with a corrosion-inhibiting primer (PAAP). Analyses are for the unbonded surface and joints exposed to liquid water.

\begin{tabular}{|l|c|c|c|c|c|c|c|c|c|c|}
\hline \multirow{2}{*}{ Specimen } & \multicolumn{9}{|l|}{ Surface composition/atomic \% } \\
\cline { 2 - 12 } & $\mathrm{C}$ & $\mathrm{O}$ & $\mathrm{Al}$ & $\mathrm{Mg}$ & $\mathrm{N}$ & $\mathrm{Ca}$ & $\mathrm{S}$ & $\mathrm{Si}$ & $\mathrm{Cu}$ & $\mathrm{P}$ \\
\hline Control Al PAAP & 35.6 & 45.0 & 18.1 & 0.3 & 0.3 & 0 & 0 & 0.2 & 0.1 & 0.4 \\
\hline Water; Metal Side & 75.3 & 20.0 & 0.2 & 0 & 4.1 & 0.2 & trace & trace & 0 & 0.2 \\
\hline Water; Adhesive & 75.4 & 19.6 & 0.5 & 0 & 3.0 & 0.2 & 0.3 & 0.7 & 0 & 0.3 \\
\hline
\end{tabular}

Table 6. Estimations of the types of loci of failure for the adhesive joints.

\begin{tabular}{|c|c|c|}
\hline Pretreatment & Test in 55\% RH & Test in Liquid Water \\
\hline GBD & $\begin{array}{l}76 \% \text { interfacial } \\
16 \% \text { cohesive in adhesive } \\
8 \% \text { cohesive in oxide }\end{array}$ & $\begin{array}{l}90 \% \text { interfacial } \\
5 \% \text { cohesive in adhesive } \\
5 \% \text { cohesive in oxide }\end{array}$ \\
\hline PAA & $100 \%$ cohesive in adhesive & $\begin{array}{l}\text { Region I } \\
70 \% \text { cohesive in adhesive } \\
30 \% \text { interfacial and cohesive } \\
\text { in oxide } \\
\text { Region II } \\
95 \% \text { interfacial } \\
5 \% \text { cohesive in oxide }\end{array}$ \\
\hline PAAP & $100 \%$ cohesive in adhesive & $\begin{array}{l}>95 \% \text { cohesive in adhesive } \\
<5 \% \text { cohesive in oxide }\end{array}$ \\
\hline
\end{tabular}




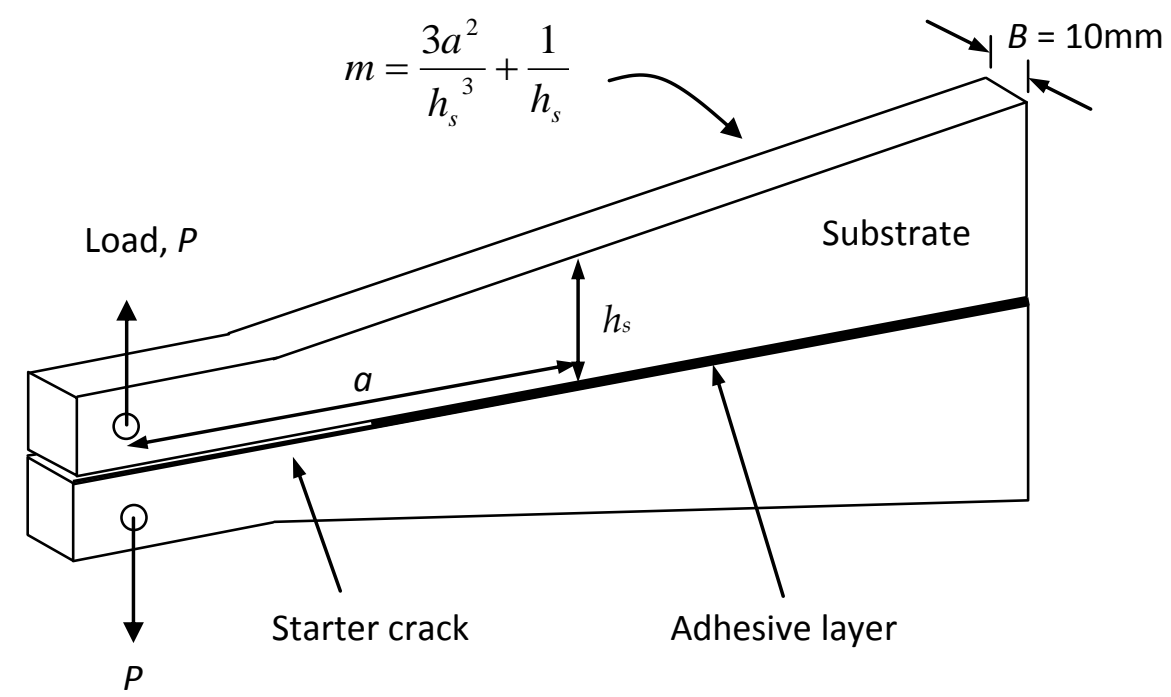

Figure 1. Schematic representation of the TDCB adhesive joint assembly. The beam is contoured in such a way that the geometrical term $m$ is constant consequently the rate of change of compliance with crack growth is constant. 


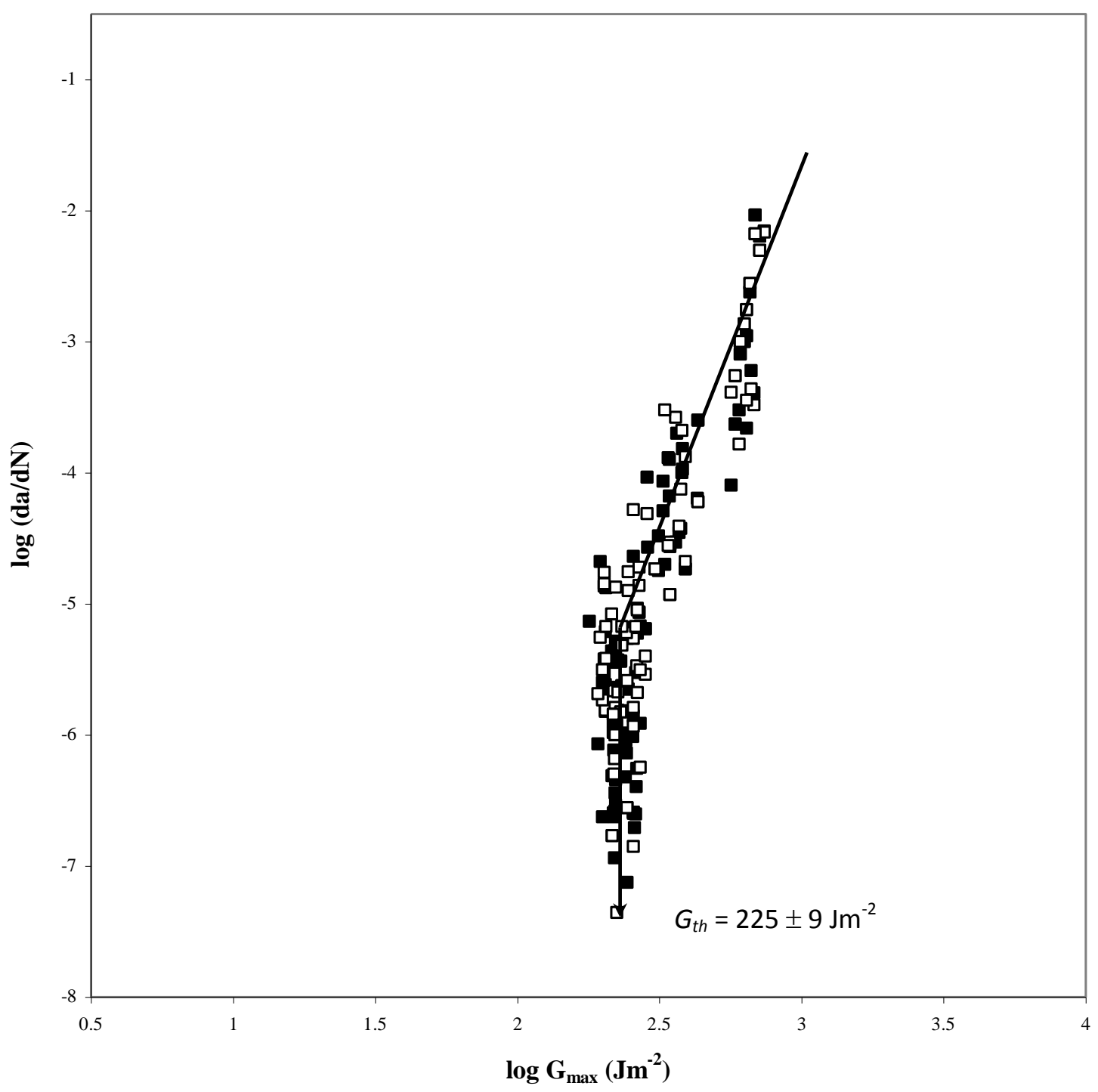

Figure 2. Log da/dN versus $\log G_{\max }$ for the PAA pretreated aluminium-alloy joints. tested at $21 \pm 1^{\circ} \mathrm{C}$ at $55 \% \mathrm{RH}$. Filled symbols represent the experimentally-measured $\mathrm{da} / \mathrm{dN}$ values; unfilled symbols represent the theoretically-determined da/dN values. 


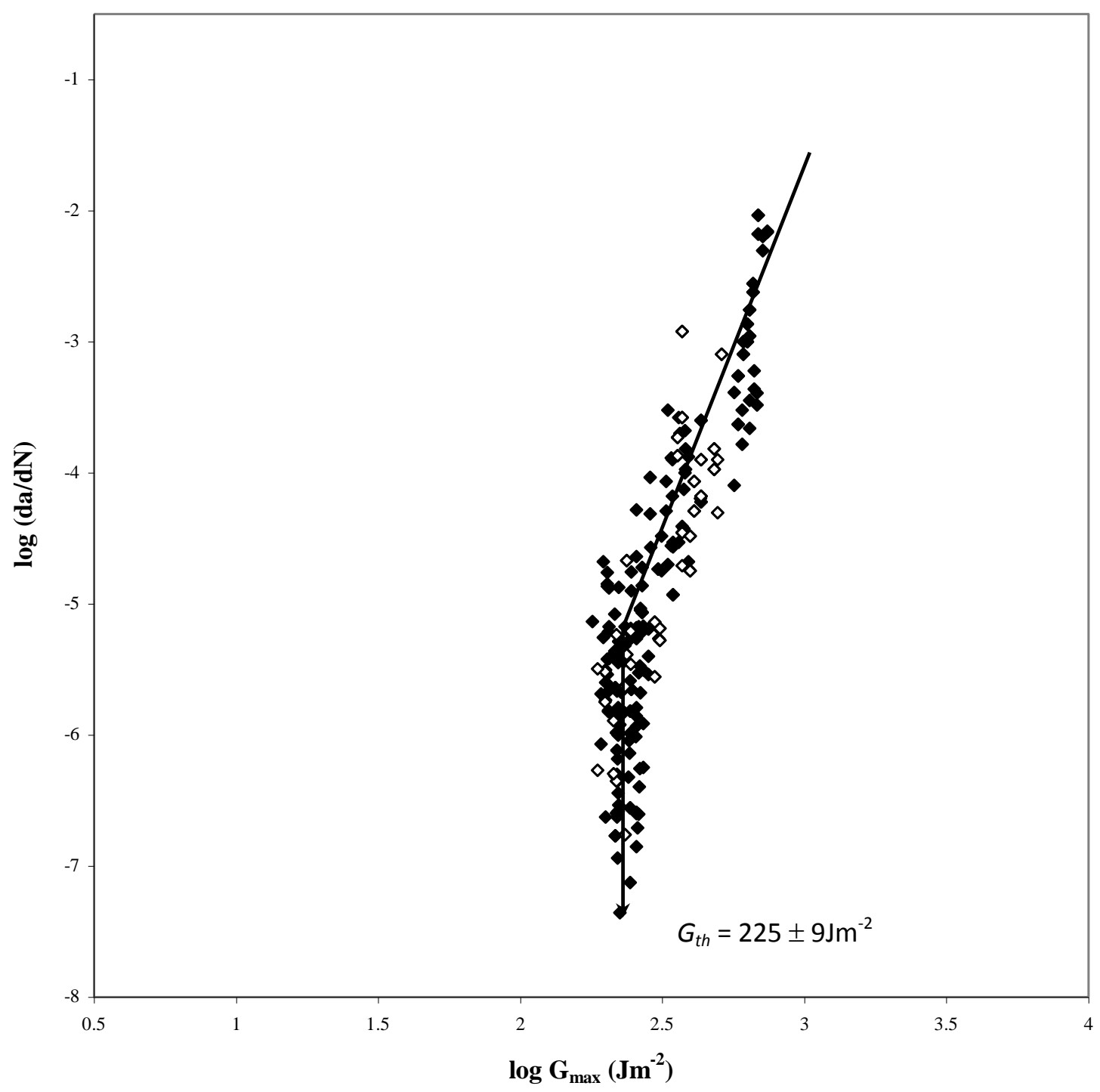

Figure 3. Log da/dN versus $\log G_{\max }$ for the PAA and PAAP pretreated aluminiumalloy joints tested at $21 \pm 1^{\circ} \mathrm{C}$ at $55 \% \mathrm{RH}$. Filled symbols represent the fatigue data for PAA pretreated joints; unfilled symbols represent the fatigue data for PAAP pretreated joints. 


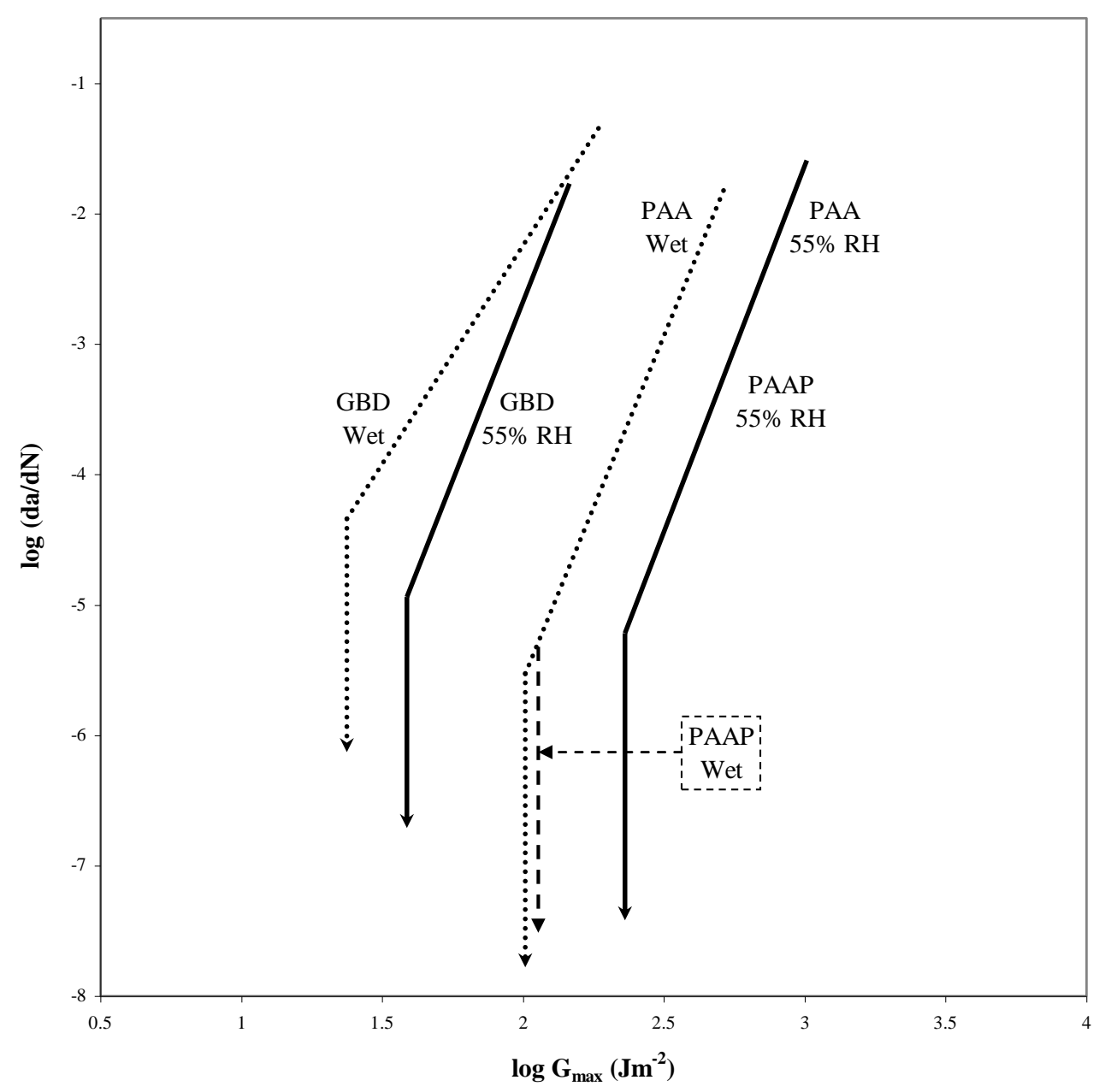

Figure 4. Log da/dN versus $\log G_{\max }$ for the GBD pretreated, PAA pretreated and PAAP aluminium-alloy joints at tested at $21 \pm 1^{\circ} \mathrm{C}$ in $55 \% \mathrm{RH}$ (solid line) and in water (dotted/dashed lines). Data points have been omitted to aid the reader, but typical scatter can be seen in the data sets of Figures 2 and 3. 


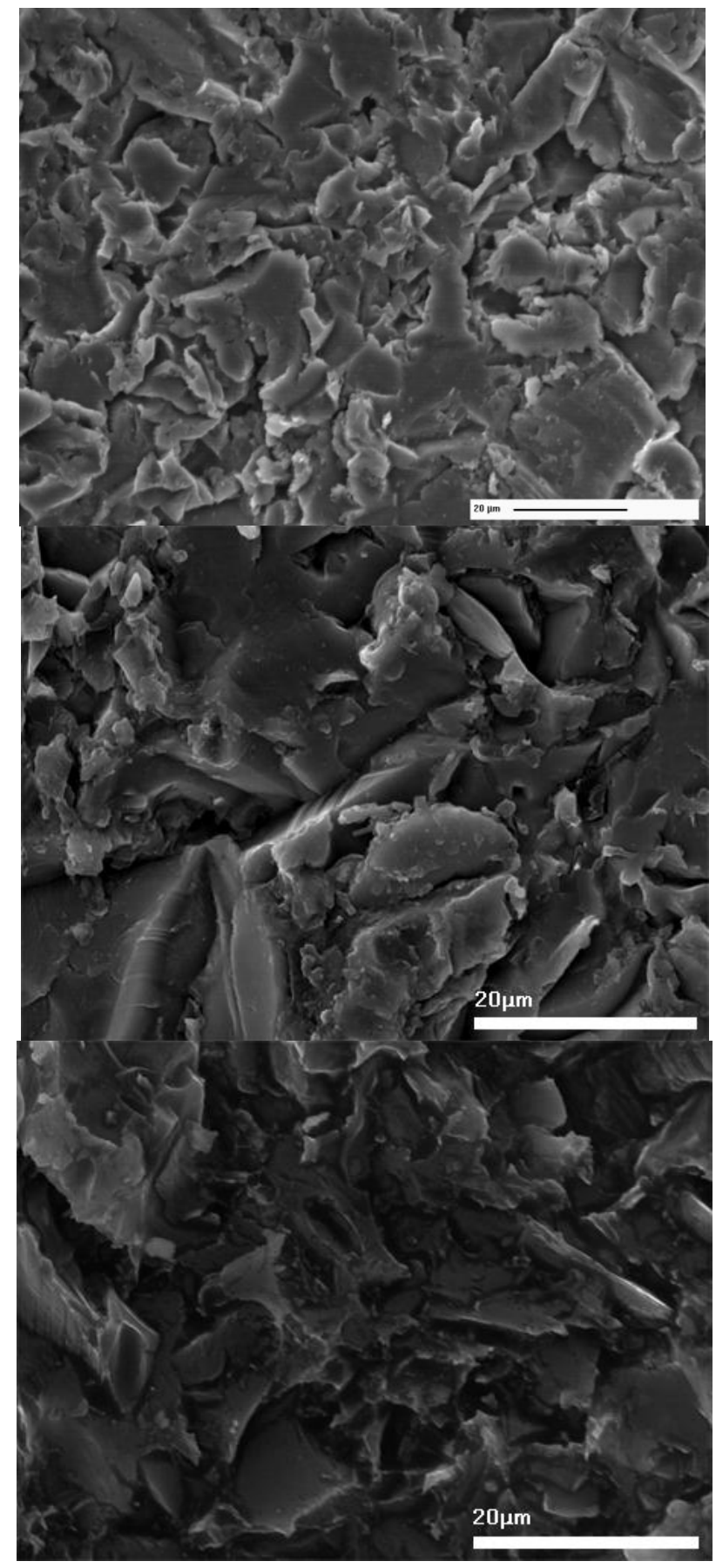

Figure 5. SEM images of the GBD pretreated aluminium-alloy substrate following (top to bottom): pretreated prior to bonding; joints tested in $55 \% \mathrm{RH}$; joints tested in liquid water. 

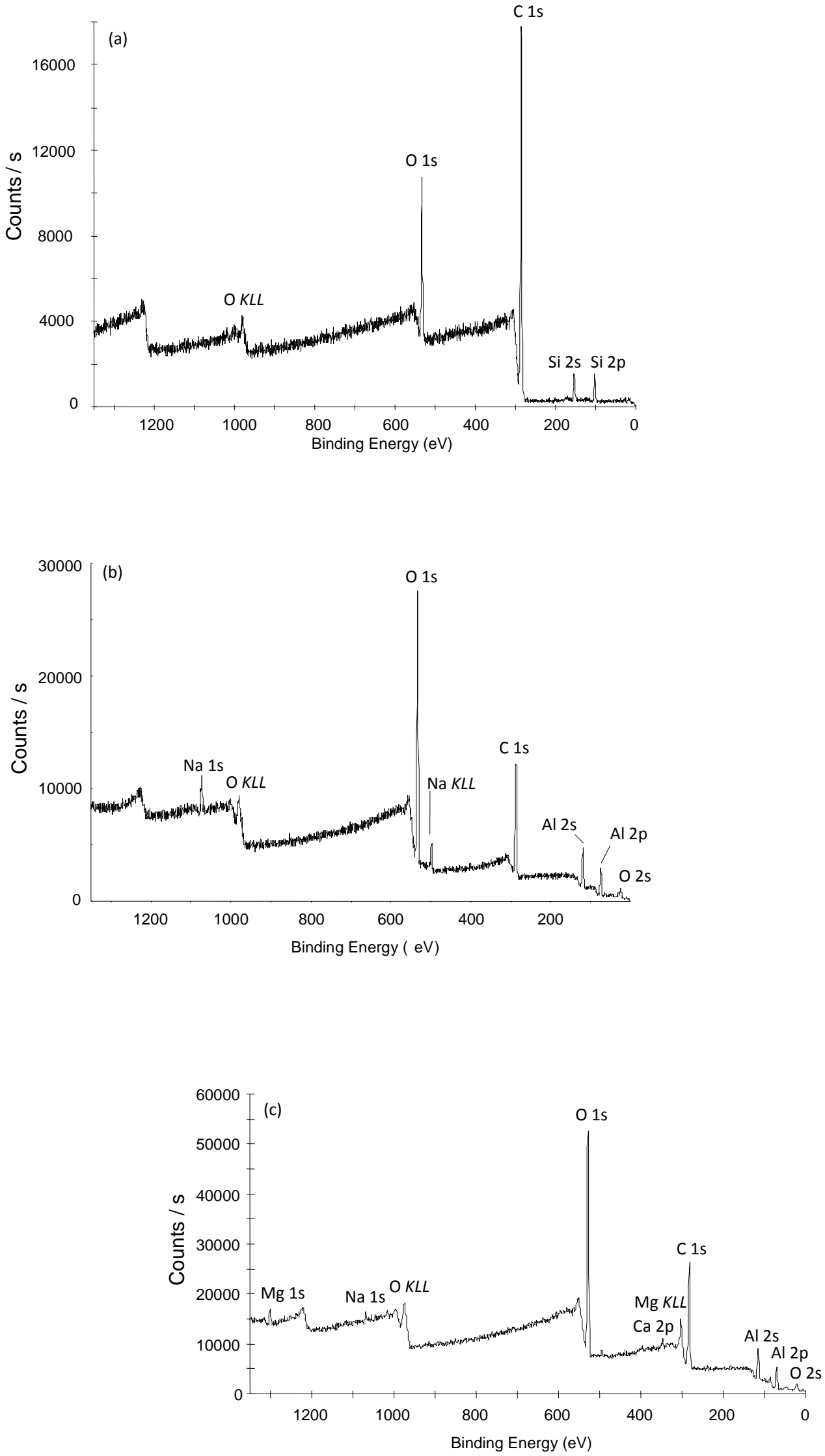


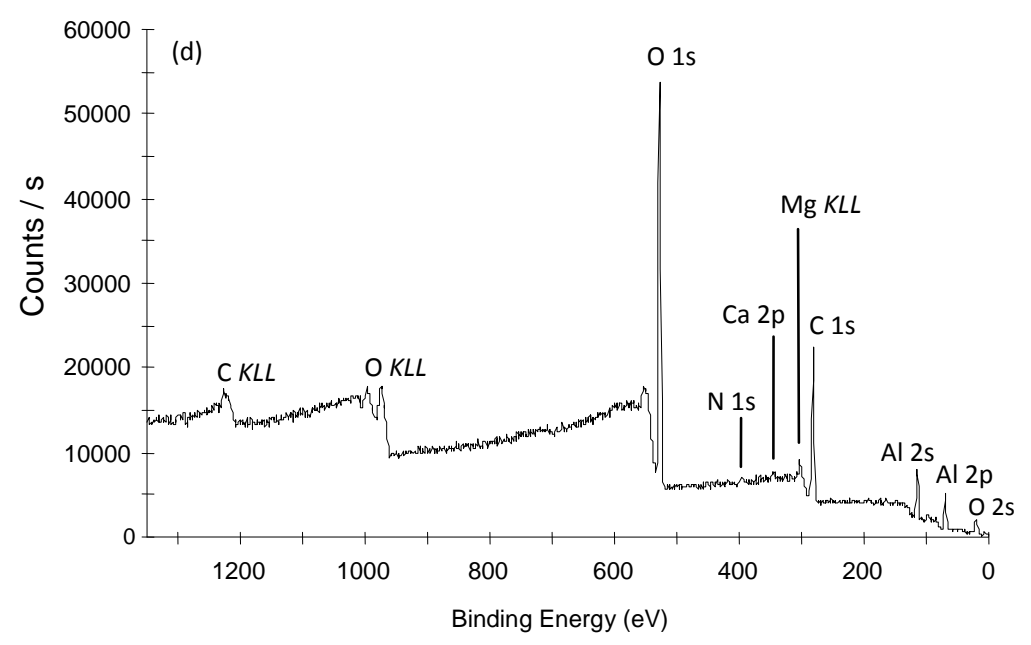

Figure 6. (a) XPS survey spectrum of adhesive, (b) XPS survey spectrum of the GBD pretreated aluminium-alloy substrate prior to bonding. XPS survey spectra of the GBD interfacial failure surfaces from, (c) joint tested in $55 \% \mathrm{RH}$, and (d) joint tested in liquid water. 

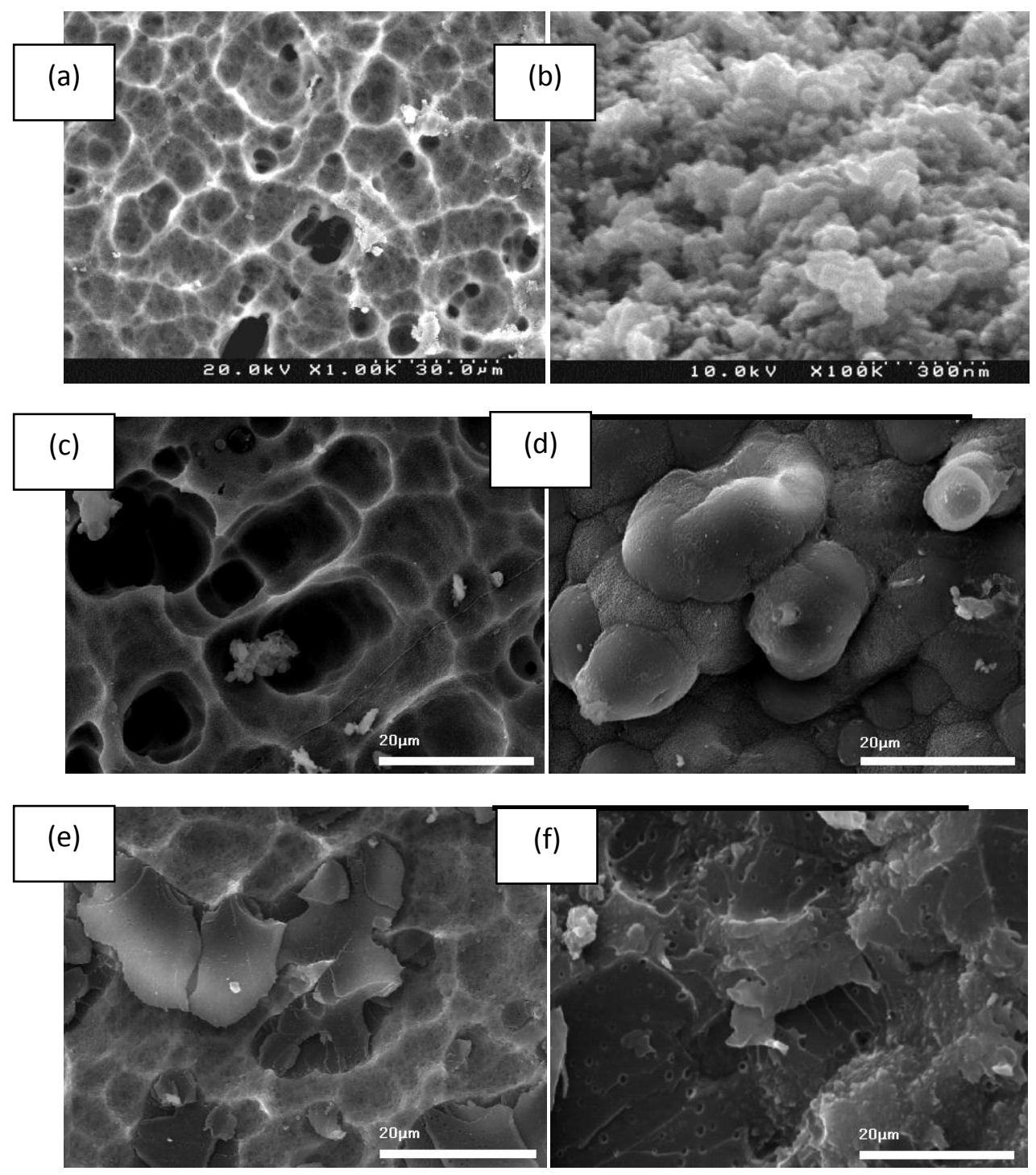

Figure 7. SEM images of PAA pretreated aluminium-alloy adhesive joint tested in water: (a) and (b) pretreated substrate prior to bonding; (c) Region II substrate surface; (d) Region II adhesive surface; (e) Region I substrate surface; (f) Region I adhesive surface. 

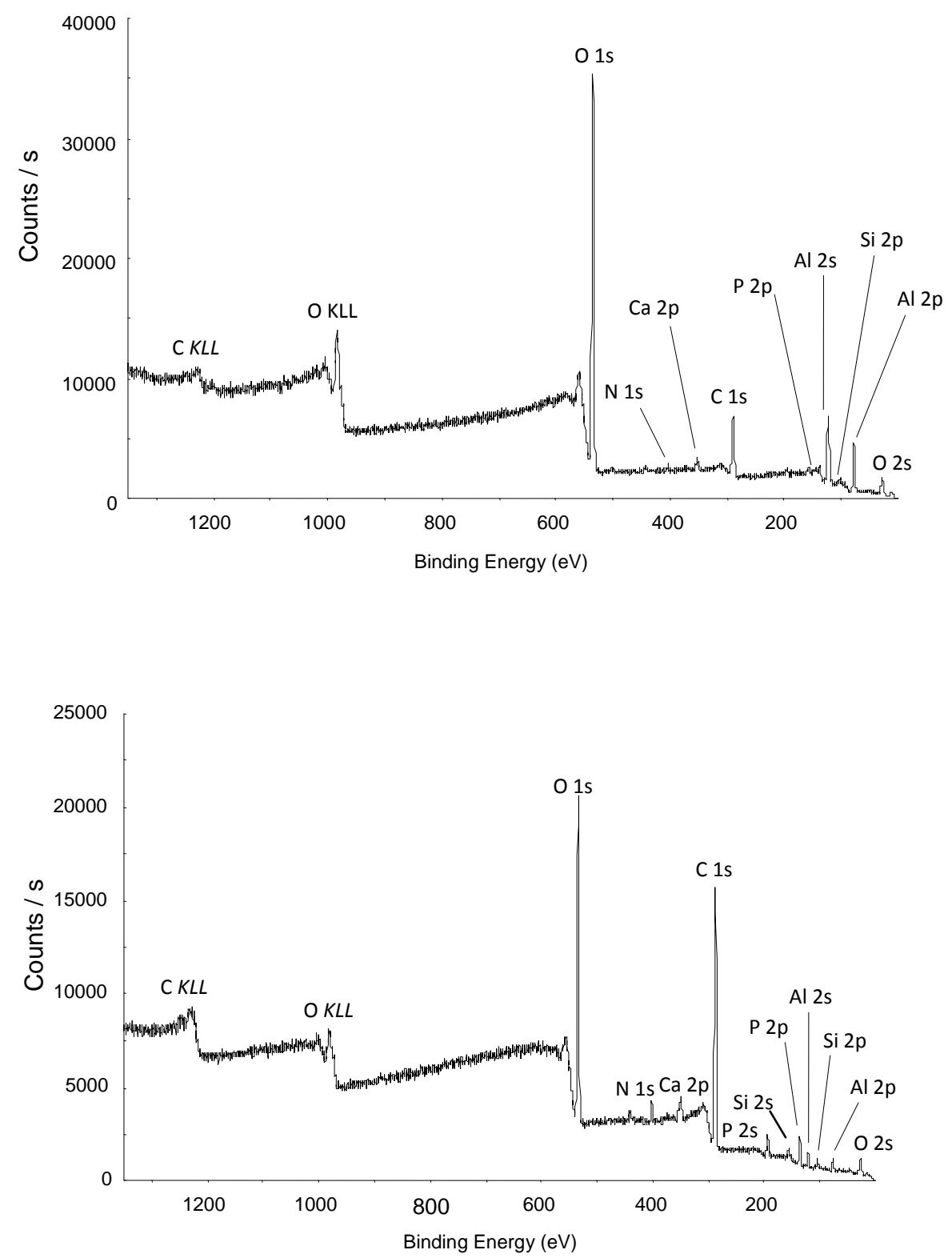

Figure 8. XPS survey spectra of the PAA treated aluminium-alloy substrates: pretreated prior to bonding (top) and from the failure surface of the joints from Region I of the crack growth curve when tested in water (bottom). 

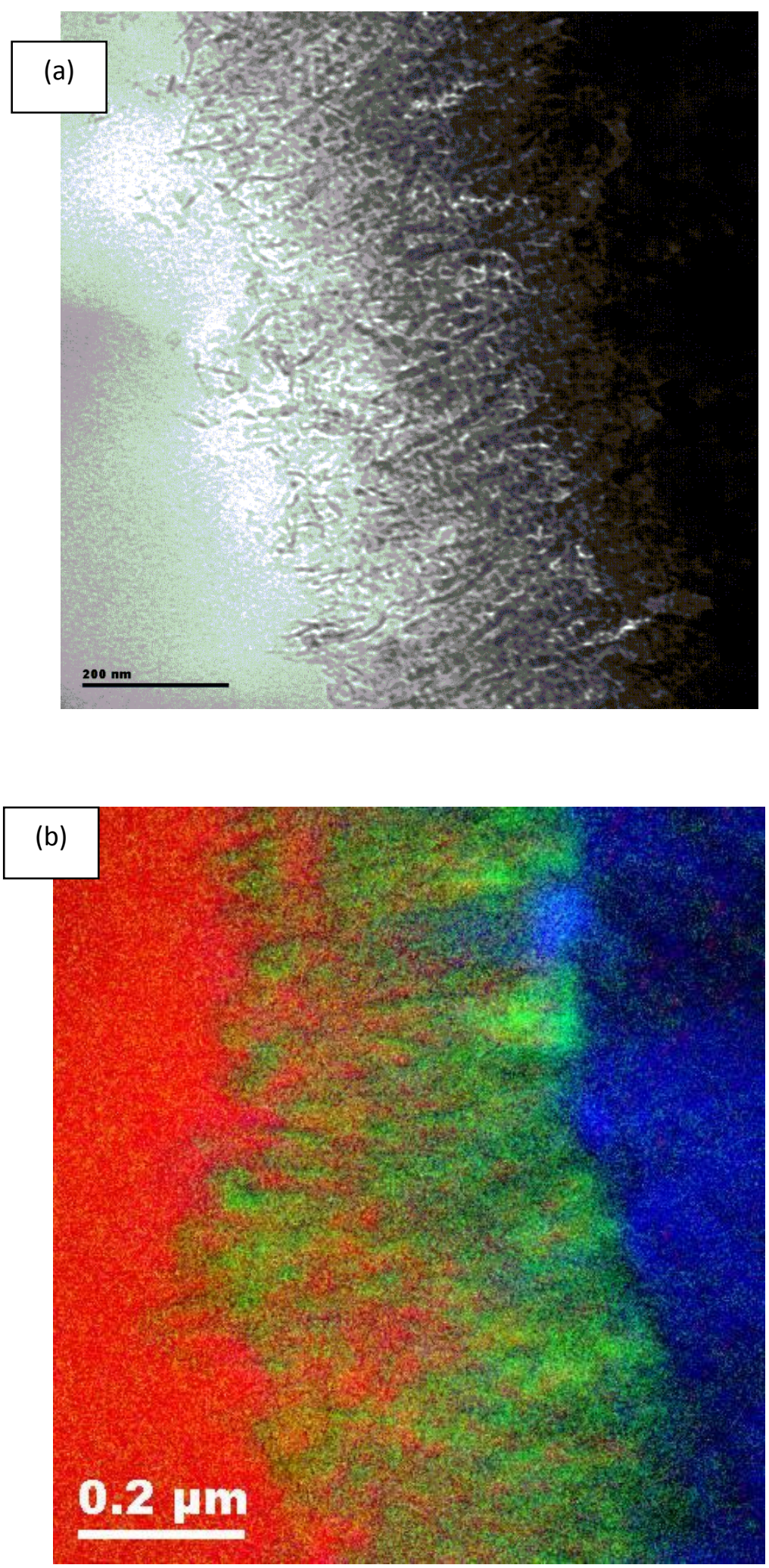

Figure 9. TEM micrograph (a) and EFTEM (PEELS) image (b) of the intact Al/PAA/adhesive interface. In the EFTEM image (b) then blue represents aluminium, green oxygen (of the PAA layer) and red carbon (of the adhesive). The interdiffusion of the adhesive into the porous PAA oxide layer is clearly evident, to form a 'microcomposite' interphase region. 

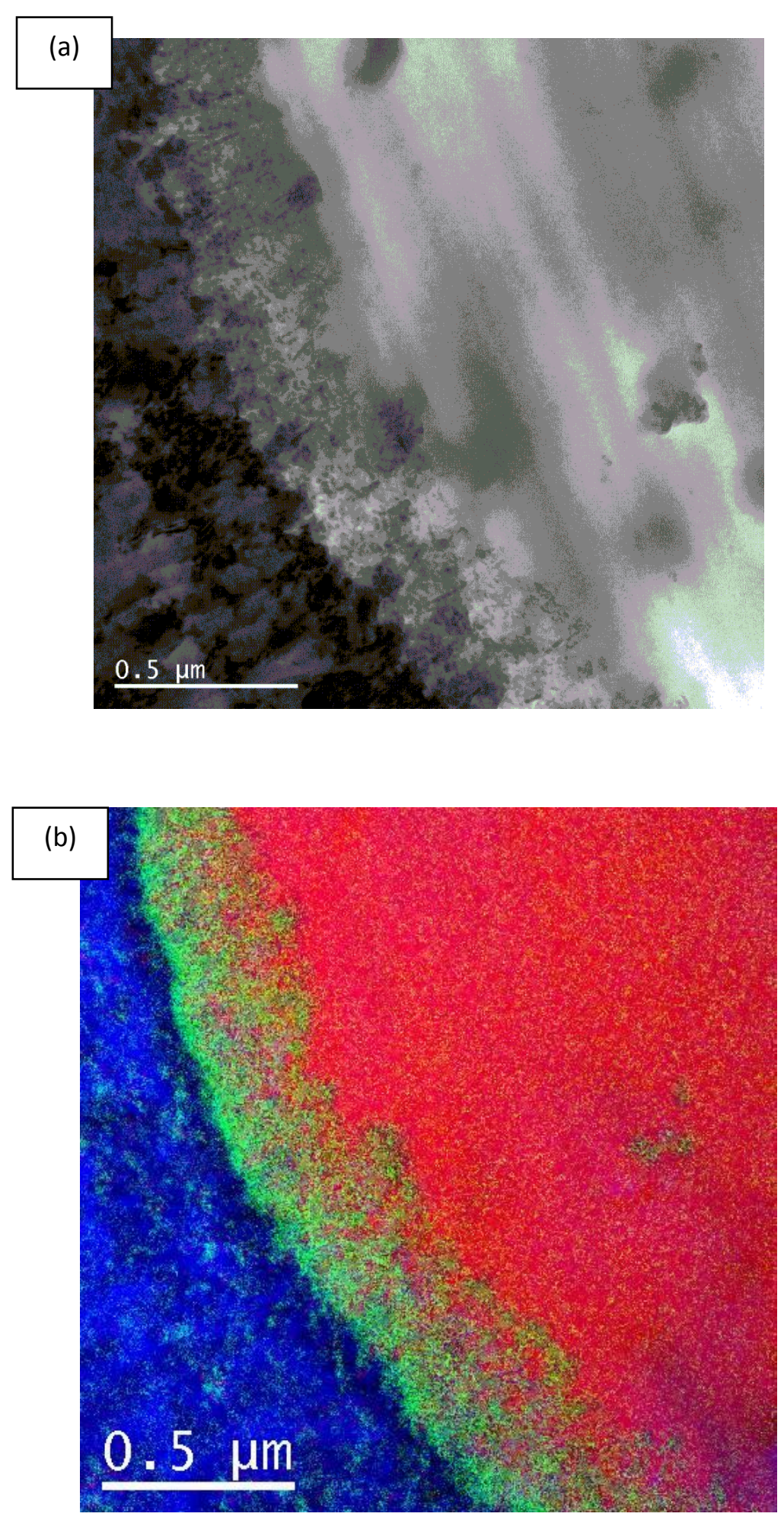

Figure 10. TEM micrograph (a) and EFTEM image (b) of a section of intact interface just ahead of the crack tip from a PAA aluminium-alloy specimen tested in water. In Figure 10(b): red represents the element carbon which is present in the adhesive; green represents oxygen in the oxide layer; and blue represents aluminium in the substrate. 


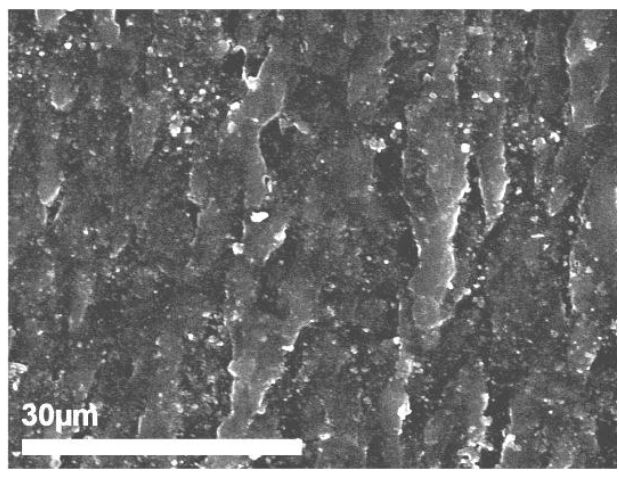

Figure 11. SEM image of the PAAP treated aluminium surface prior to bonding.

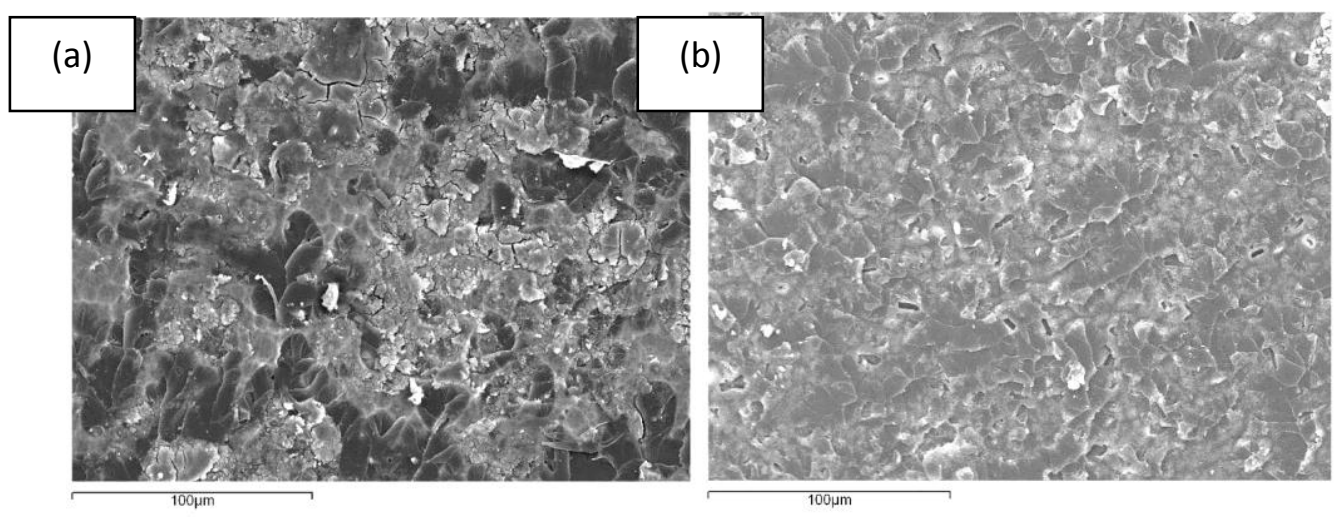

Figure 12. SEM images of PAAP pretreated joint: (a) metal and (b) adhesive failure surfaces of a specimen taken from the cyclic-fatigue threshold growth zone, tested in water. 

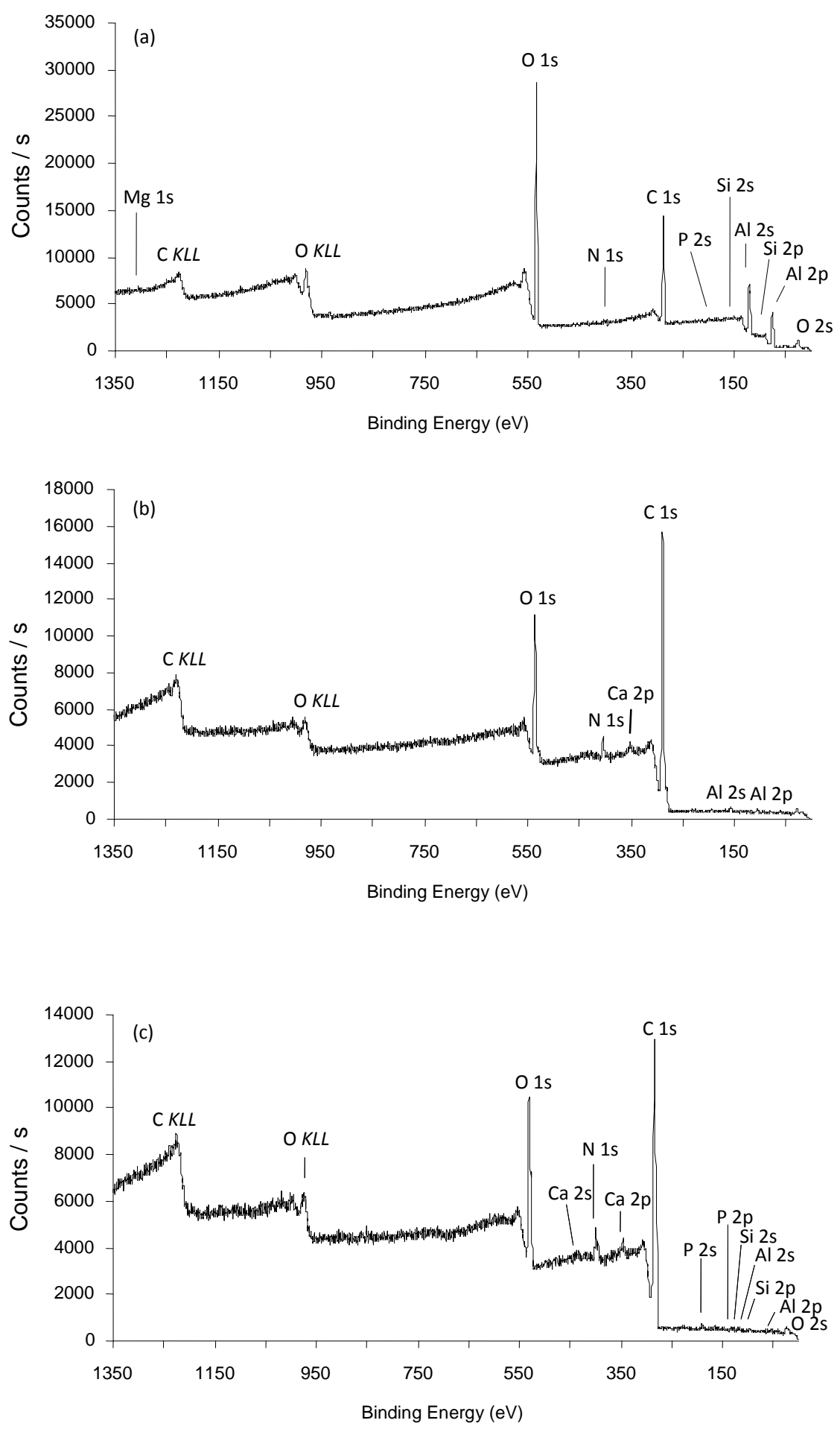

Figure 13. PAAP pretreated aluminium-alloy adhesive joints. (a) Pretreated surface, (b) joint metal failure surface and (c) joint adhesive failure surface. 

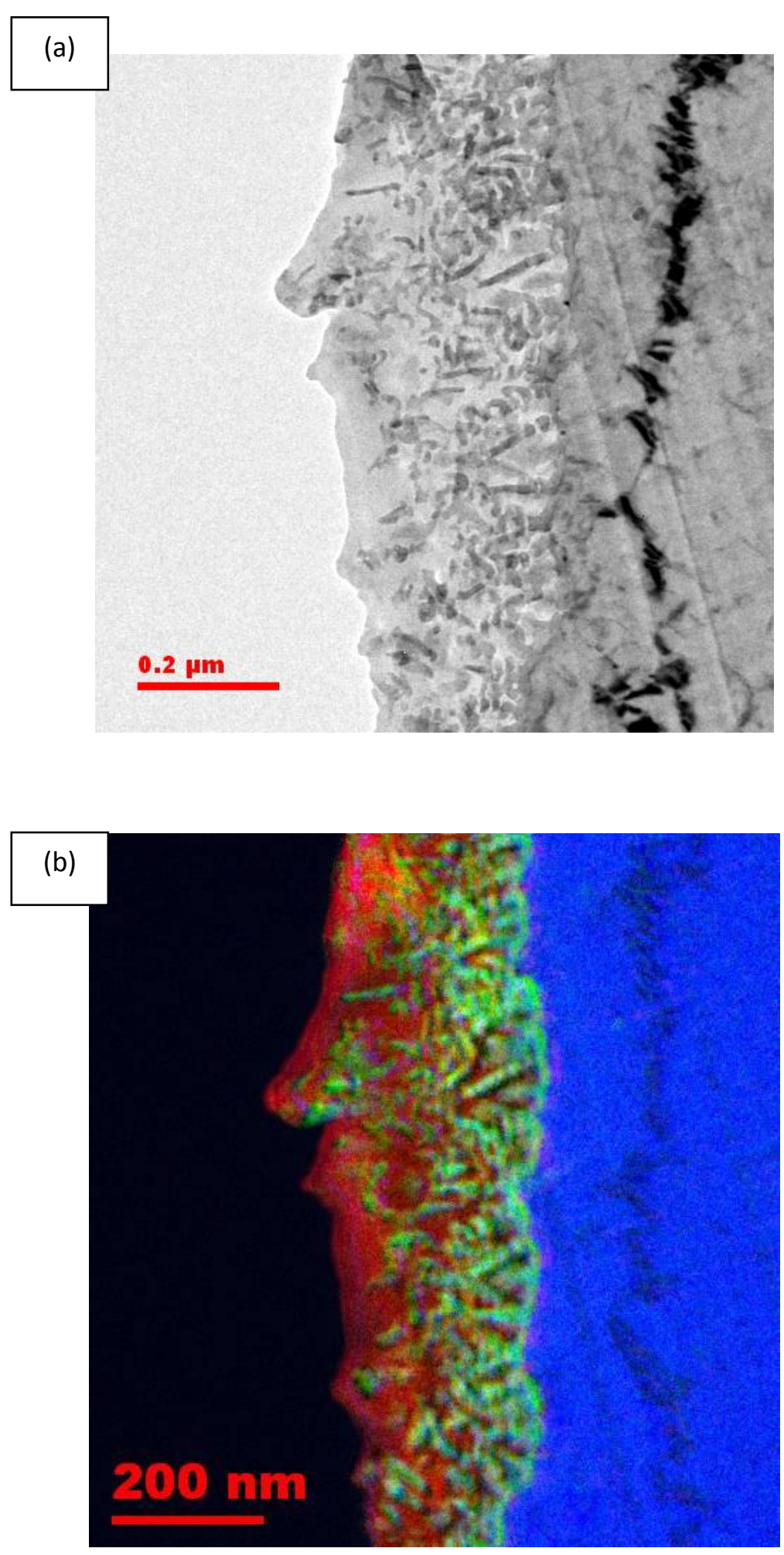

Figure 14. (a) TEM and (b) EFTEM images from the metal substrate side of a failed PAAP adhesive joint, tested in water. In Figure 14(b): red represents the element carbon which is present in the adhesive; green represents oxygen in the oxide layer; and blue represents aluminium in the substrate. 


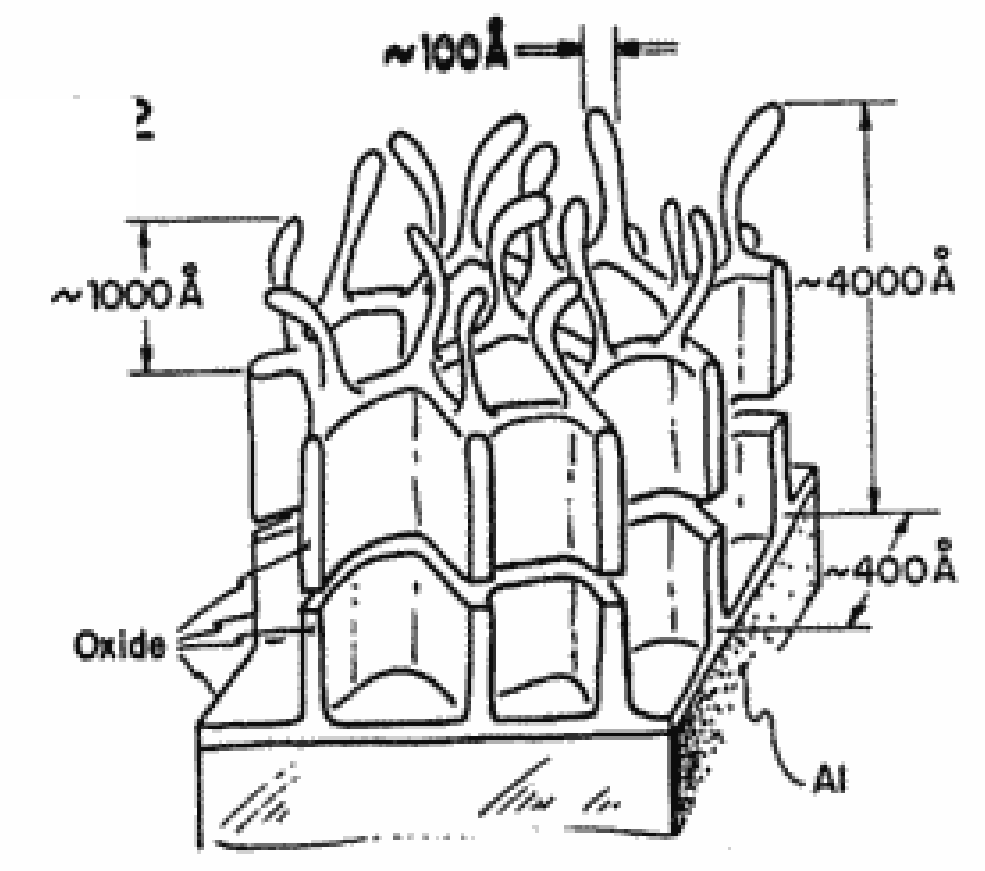

Figure 15. Schematic representation of the oxide morphology produced by phosphoric acid anodising (PAA). Note the characteristic porous hexagonal oxide structure and "finger-like" protrusions emanating from the hexagonal structure. Reproduced from Venables (18) courtesy of Springer. N.B. $10 \AA=1 \mathrm{~nm}$. 